\title{
1. Stochastic game theory for social science: a primer on quantal response equilibrium*
}

\author{
Jacob K. Goeree, Charles A. Holt and Thomas R. Palfrey
}

\section{INTRODUCTION: QUANTAL RESPONSE EQUILIBRIUM (QRE) AS A GENERALIZATION OF NASH EQUILIBRIUM}

Most people, with a few prominent exceptions, will admit to making mistakes. Moreover, it is apparent from analysis of competitive situations in athletics, business and politics that people may alter their behavior in anticipation of their own and others' mistakes. In contrast, standard game theory is based on the assumption that players are perfect maximizers; that is, they use their beliefs about uncertain events, including other players' actions, to make decisions that are consistent with the maximization of expected payoffs. An equilibrium results if there are no incentives for learning or change. Thus the initial beliefs about others' decisions must be consistent with those decisions, at least in a probabilistic sense.

The idea of modeling imperfect maximization with stochastic choice derives from the work of mathematical psychologists, such as Thurstone (1927) and Luce (1959). This work was motivated by choice experiments in which subjects were asked to assess the relative intensities of two lights or two sounds. Observed choice frequencies tended to be correlated with the difference in the stimulus intensity, but with some randomness when the intensity differences were small. The simplest Luce model stipulates that the choice frequency is the ratio of the stimulus intensity for that choice to the sum of intensities for all possible choices. In economic models, the stimulus can be measured in expected payoffs. Suppose that there are two decisions, $D 1$ and $D 2$, with expected payoffs denoted by $\pi_{1}$ and $\pi_{2}$, so that the Luce ratio rule would be that the probability of $D 1$ is $\operatorname{Pr}(D 1)=\pi_{1} /\left(\pi_{1}+\pi_{2}\right)$. This rule exhibits an important responsiveness property in that the probability of choosing a decision is an increasing function of the expected payoff for that decision. Notice that if the stimuli are of equal intensity, then the choice probability is 0.5 , although other choice functions with this intuitive symmetry property can be used.

In QRE theory, the basic building block for stochastic choice is the quantal response function (McKelvey and Palfrey 1995, 1996, 1998; Goeree et al. 2016), of which the Luce ratio rule is a special case. Consider a simple symmetric game with two players, each with two decisions. In this game, the expected payoffs could be functions of a belief $p$ that represents a player's beliefs about the likelihood that the other player chooses $D 1$, so the Luce quantal response function would specify:

$$
\left.\operatorname{Pr}(D 1)=\frac{\pi_{1}(p)}{\pi_{1}(p)+\pi_{2}(p)} \text { (Luce ratio probabilistic choice rule }\right) .
$$

The probabilistic choice function is a quantal response, in contrast with a best response that would imply a probability 1 associated with the decision with higher payoffs. A quantal response equilibrium in this symmetric context would require that the $p$ representing beliefs in 
the expected payoffs on the right-hand side of (1.1) be equal to the choice probability, $\operatorname{Pr}(D 1)$, that is determined by the quantal response function. In equilibrium, there is no opportunity for learning in that the quantal response probabilities match the beliefs, that is, $\operatorname{Pr}(D 1)=p$. For example, suppose there is congestion in location 1 in that $\pi_{1}(p)=V-p$, with $V>1$, whereas there is no congestion in location $2: \pi_{2}(p)=0.5$. In this example, $\operatorname{Pr}(D 1)=p=$ $(V-p) /(V-p+0.5)$. The resulting equilibrium choice probability is increasing in $V$. For example, $p=0.5$ when $V=1$, which increases to $p=2 / 3$ when $V=5 / 3$.

The Luce quantal response function is useful in some applications, but it has two drawbacks. First, it is only defined for positive payoffs and would need to be adjusted if payoffs can be negative, in order to ensure non-negative choice probabilities. We might think that simply adding a sufficiently large constant to each payoff so that all payoffs are positive would solve the problem. Unfortunately, and this is the second drawback of using the Luce model as a quantal response function: we cannot arbitrarily add constants to payoffs in the Luce choice model, as the addition of a constant will change all of the choice probabilities. ${ }^{1}$ Another useful feature is to introduce a parameter that determines the amount of noise in the probabilistic choice, so that the limiting case of no noise (best responses) can represent the decision rule used in a Nash equilibrium in which each player best responds to the other's choices. One way to handle both issues - negative payoffs and inclusion of a response parameter - is to replace the expected payoffs with exponential functions parameterized by precision, $\lambda>0:^{2}$

$$
\operatorname{Pr}(D 1)=\frac{\exp \left(\lambda \pi_{1}(p)\right)}{\exp \left(\lambda \pi_{1}(p)\right)+\exp \left(\lambda \pi_{2}(p)\right)}=\frac{1}{1+e^{-\lambda x}} \text { where } x=\pi_{1}(p)-\pi_{2}(p) \text { (Logit). }
$$

The logit quantal response function in (1.2) is positively responsive to payoffs. Moreover, it is defined for both positive and negative payoffs, is strictly positive for all actions and it satisfies symmetry, since the choice probability is 0.5 when the expected payoffs are equal, or equivalently, when the payoff difference $x$ is 0 . Figure 1.1 shows the logit probabilistic choice rule as a function of the expected payoff difference on the horizontal axis. The flatter curve with the $S$ shape was constructed with a precision of $\lambda=0.2$, whereas the sharper curve was generated with a high precision of $\lambda=2$. The high-precision, sharp function puts a probability of almost 1 on decision $D 1$ when it has the higher payoff, that is, when $\pi_{1}(p)-\pi_{2}(p)>0$. The logit rule has long been widely applied in economics in the analysis of individual decisions, for example, the choice of a commuting route (McFadden 1976), and it is now commonly used in the analysis of QRE for games that are implemented in laboratory experiments.

Quantal response equilibrium preserves the underlying structure of classical game theory, but adds stochastic choice to the decision-making process. This modification is implemented in a manner ensuring that smaller, relatively inconsequential errors are more likely than costly errors. Expected payoff-maximizing best responses are replaced by better responses. A QRE is a fixed point of quantal response functions, just as Nash equilibrium is a fixed point of best response functions. Hence the distributions representing players' beliefs are consistent with the quantal responses to those beliefs. A fixed point theorem was used to prove existence in the original paper on QRE by Richard McKelvey and Thomas Palfrey, published in Games and Economic Behavior in 1995. Thus QRE is a generalization of the standard notion of Nash 


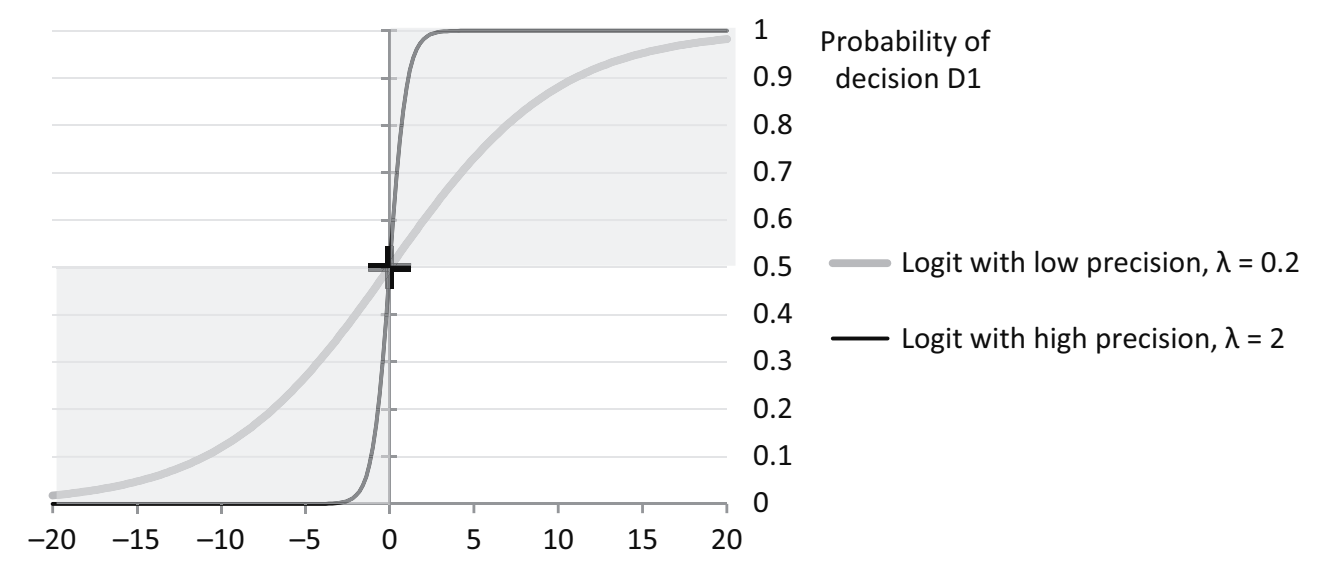

Expected payoff difference $\pi_{1}-\pi_{2}$ for choosing decision $D 1$

Notes: The flatter curve is for low precision, $\lambda=0.2$, where the sharper function is for high precision $\lambda=2$. Notice that both functions pass through the midpoint $(x=0, p=0.5)$, as required by symmetry.

\section{Figure 1.1 Logit probabilistic choice for decision D1}

equilibrium, and it can be shown that quantal response equilibria converge to Nash equilibria as noise is diminished at the limit (see McKelvey and Palfrey 1995, theorem 2; Goeree et al. 2016, ch. 1).

A natural question to ask is, why is generalization necessary? After all, the standard analysis of equilibrium in games, which began with the seminal work of von Neumann and Morgenstern (1944) and Nash (1950), has been extended to include restrictions on rational choice in dynamic settings (Selten 1965) and stochastic effects caused by privately known individual differences (Harsanyi 1973). ${ }^{3}$ Game theory has provided important insights for the development of new auction and mechanism designs (Vickrey 1961; Roth 1992), and for the analysis of issues of industrial organization, regulation and public policy (Tirole 1988). Applications to other fields, such as political science and law, are extensive. Indeed, a great deal of the early work in the mid-twentieth century was motivated by (and funded to deal with) bargaining between nation states in the shadow of conflict. Political scientists have developed a variety of useful paradigms, for example, the Baron and Ferejohn (1989) legislative bargaining game and Elinor Ostrom's (1990) analysis of common pool resource games in small societies. Game theory and its behavioral extensions currently constitute the closest thing there is to a unifying theory of social science.

Although game theory has been used to structure empirical work using naturally occurring data, most careful testing of the Nash equilibrium and related theory has involved controlled experiments, either in the laboratory (for example, Selten and Stoecker 1986; Roth 1995) or in the field (Ostrom et al. 1994). Here the message is mixed. One of the most widely used paradigms in the social sciences, the prisoner's dilemma, is a model that had its beginnings in a 1950 laboratory experiment designed to show that the equilibrium prediction (to defect) is not a likely outcome with repeated plays of the game. ${ }^{4}$ Moreover, Nash recognized that bargaining theory was not very useful in studying negotiations in the laboratory (there was no explicit consideration of fairness or inequity aversion in those days). Selten's 
path-breaking theoretical work on subgame perfection was initially a response to the need to eliminate the many irrelevant Nash equilibria for an oligopoly model used in one of his early laboratory experiments. Even with subgame perfection, results of simple ultimatum bargaining experiments are sharply at odds with theoretical predictions (Güth et al. 1982; and as described by Selten in Svorenčík and Mass 2016, pp. 155-6).

The failures of subgame perfection are apparent for a centipede game, which was devised by Rosenthal (1982) to demonstrate the implausibility of the backward induction rationality in the presence of a large number of stages. The centipede game involves passing a tray with increasing and unequal payoffs back and forth between two players until one of them takes, which implements the payoffs at that point. Payoffs are augmented with each pass, but the relative payoffs are reversed. For example, the initial payoffs of $\$ 4$ for $A$ and $\$ 1$ for B in the first stage are changed to $\$ 2$ for $A$ and $\$ 8$ for B in the second stage, and so on. Thus A should take at the first stage if $\mathrm{B}$ is expected to take at the second stage. With a finite, known number of stages, a process of backward induction implies that A take at the very first stage and stop the game at that point. In contrast, take decisions at the very first stage are uncommon (less than 10 percent) in laboratory experiments, even with repeated random matching (McKelvey and Palfrey 1992). ${ }^{5}$

Theorists have long recognized that an overhaul of game theory is needed, but the news from controlled experiments is not uniformly bad, since observed patterns suggest some important features that should be captured by a more behaviorally relevant theory. Human subjects typically do show systematic correlations between observed behavior and changes in payoff incentives, although central tendencies can be far from the mark in some cases. In centipede experiments with potential payoffs in thousands of dollars, for example, take rates are increased, although take rates near the predicted probability of 1 in the first stage are still not the norm (Parco et al. 2002). So theory should be responsive, that is, sensitive to payoff differences. Moreover, the noise in the data is inconsistent with point predictions that emerge from simple game-theoretic models, which highlights the need for a statistical theory that assigns positive probabilities, however small, to all outcomes.

Quantal response equilibrium theory handles interactions by incorporating probabilistic choice functions into the equilibrium analysis of games. The frequencies determined by these quantal choice models show some spread that depends on the balance between incentives (differences in stimuli) and noise - owing either to bounded rationality or to unobserved latent variables, which is the more common view in economic applications. Regardless of the source, the resulting distribution around central tendencies in simple decision problems can be magnified owing to cascading effects that shift the entire choice distribution in games with interdependent payoffs. Quantal response equilibrium builds on Harsanyi's (1973) path-breaking work on games with incomplete information, by letting agent-specific shocks represent the effects of the latent variables that inject noise into the system. This approach was also used in the theoretical analysis of learning in games (Fudenberg and Kreps 1993). The specific nature of the distributions of payoff disturbances, for example, logit or probit, results in game-theoretic QRE models that are natural generalizations of widely used logit and probit econometric models of individual decisions.

If payoffs are perceived with error, which can be modeled by adding epsilon errors to actual payoffs, then one issue is whether the added flexibility provided by error specifications provides so much flexibility that the model has no empirical content. It is essential that a theory that permits deviations from perfect rationality maintains a degree of payoff 
responsiveness that preserves predictive power. This question was raised by Haile et al. (2008):

The quantal response equilibrium (QRE) notion of Richard D. McKelvey and Thomas R. Palfrey (1995) has recently attracted considerable attention, due in part to its widely documented ability to rationalize observed behavior in games played by experimental subjects ... However, even with strong a priori restrictions on unobservables, QRE imposes no falsifiable restrictions: it can rationalize any distribution of behavior in any normal form game.

Their argument is based on QRE in its most general form, for which an existence proof does not require that payoff disturbances be independent and identically distributed. It is easy to understand how this can happen if random disturbances are not independent, for example, if the random payoff disturbance associated with one decision is systematically higher than that for another, even though both distributions have a zero mean. For example, suppose that decisions $D 1$ and $D 2$ have payoffs of 1 and 2 (a difference of 1 ), but the disturbance for the high-payoff decision $D 2$ is always 0 , whereas the disturbance realization for the low payoff decision $D 1$ is 2 with probability $p$, except on a correction interval of size $1-p$, where the disturbance realization for $D 1$ is an amount, $-x$. The magnitude of $x$ is chosen to ensure that the expected value of that disturbance equals 0 . With disturbances that are not identically distributed in this sense, the choice probabilities for inferior decisions can be enhanced to any desired extent. A similar argument can be made when the disturbances are not independently distributed. These counterintuitive results are not possible with independent and identically distributed (i.i.d.) disturbances. ${ }^{6}$ The empirical content critique is irrelevant in that standard assumptions (i.i.d. disturbances) are sufficient to ensure that the QRE can make strong predictions, even for an experiment with a single treatment (examples to follow). ${ }^{7}$ Empirical restrictions are even stronger when an experiment has multiple treatments and the same QRE model is used for all treatments, as is the norm. Multiple treatment designs and standard i.i.d. assumptions underlie virtually all empirical applications of QRE, just as i.i.d. logistic and normal errors are the basic components of the widely used logit and probit econometric models of individual decisions.

The basic insight about empirical content is that an i.i.d. assumption on payoff disturbances requires that the probabilistic choice function, logit, probit or anything in a wide class, will be a non-decreasing cumulative distribution function that passes through the center point of Figure 1.1. This is because when payoffs are equal (the center vertical line), the choice probability has to be one half (midpoint of the line). The probabilistic functions can be flatter or sharper as they rotate around this center point, but any QRE would be a point in one of the two shaded rectangles in Figure 1.1. That is, a QRE will be a belief probability and associated expected payoff difference point in one of these shaded rectangles. The nature of the expected payoff differences for the particular structure of a game will generate clear restrictions on the set of choice probabilities that could be QRE points, as shown in the following sections.

The next section develops the connection between distributions of additive payoff perturbations and shapes of quantal response functions, for a class of symmetric games with binary decisions. The analysis is based on a graphical device that separates payoff and noise factors, so that the fixed point involves the intersection of lines for each of these factors. This graphical representation permits an analysis of existence, comparative statics and stability issues. In subsequent sections, the analysis is illustrated for a variety of increasingly complex games, 
for example, prisoner's dilemma, battle of sexes, coordination, volunteer's dilemma, matching pennies and imperfect price competition. ${ }^{8}$

\section{THE CONNECTION BETWEEN PAYOFF PERTURBATIONS AND QUANTAL RESPONSES}

The structural approach to quantal response is based on assumptions about the nature of random, decision-specific payoff perturbations that can soften the connection between payoffs and decisions. A more reduced-form approach is to begin with assumptions about the properties of quantal response functions that map expected payoffs into choice probabilities. In this section, the close connection between these two approaches is explained in the context of simple class of symmetric binary-choice games, in which the two possible actions (or decisions) are labeled $D 1$ and $D 2$. In each example to be considered, $D 1$ refers to the more pro-social decision, for example, cooperate in a prisoner's dilemma, exert high effort in a coordination game, volunteer to provide a public good, vote, invest in security, or exit from a congested environment.

Each player has an expected payoff function that depends on the probability $p$ that the other player chooses the pro-social decision $D 1$. In a symmetric equilibrium with $N$ players, $p$ represents the probability that each of the $N-1$ others chooses $D 1$. As before, these expected payoffs will be denoted by $\pi_{1}(p)$ and $\pi_{2}(p)$ for decisions $D 1$ and $D 2$, respectively, where the symmetry precludes the need for player-specific payoff functions (extensions to asymmetric games are discussed subsequently).

Perceived or perturbed payoffs are modeled as sums of payoffs and additive (playerspecific) disturbances, $\varepsilon_{i j}$, so that a player $j$ will select $D 1$ if $\pi_{1}+\varepsilon_{1 j} / \lambda>\pi_{2}+\varepsilon_{2 j} / \lambda$, or if $\pi_{1}-\pi_{2}>\left(\varepsilon_{2 j}-\varepsilon_{1 j}\right) / \lambda$, or equivalently, if $\varepsilon_{2 j}-\varepsilon_{1 j}<\lambda\left(\pi_{1}-\pi_{2}\right)$, where the positive precision parameter $\lambda$ determines the importance of the disturbance. As $\lambda \rightarrow \infty$, the $\varepsilon_{i j} / \lambda$ terms go to zero and the effects of the random disturbances become irrelevant (perfect rationality). Recall that expected payoffs are functions of $p$, which can be thought of as representing a player's beliefs about the chances that the other(s) will choose $D 1$, and this difference will be denoted by $\Delta(p)=\pi_{1}(p)-\pi_{2}(p)$. Thus the probability of choosing $D 1$ is determined by the probability that the difference in disturbances will be less than the scaled payoff difference, $\lambda \Delta(p)$. Let $F(\cdot)$ denote the cumulative distribution function associated with the differences in disturbances, so the probability of choosing $D 1$ is the probability that $\varepsilon_{2 j}-\varepsilon_{1 j}<\lambda \Delta(p)$ can be written as $F(\lambda \Delta(p))$. In equilibrium (with no incentive for change), the choice probability on the left-hand side of (1.3) must equal the belief probability $p$ used in the payoff difference on the right:

$$
p=F(\lambda \Delta(p)) .
$$

The distribution function $F$ is assumed to be continuous and monotonically increasing on the real line. That is, the distributions have full support, so anything is possible (however unlikely), which is needed to avoid the zero likelihood problem. This full-support property is important when considering laboratory data generated by human subjects.

The disturbances are assumed to be identically and independently distributed, so each disturbance is equally likely to be larger or smaller than the other. Therefore, $F(0)=1 / 2$, 


\section{Handbook of experimental game theory}

that is, the difference in disturbances is equally likely to be positive or negative. It can also be shown that the i.i.d. assumption requires that the distribution of the difference in perturbations be symmetric, that is, that $F(x)=1-F(-x)$, so $F(0)=1 / 2$. Payoff functions are assumed to be continuous, so (1.3) is a continuous mapping from a compact set $[0,1]$ into itself, and a fixed point will exist (Brouwer's theorem). Uniqueness is another story, as is apparent from the examples provided.

If the payoff perturbations are Gaussian, for example, then their difference is Gaussian with mean zero and full support. In that case, $F(\cdot)$ represents the cumulative distribution of a Normal distribution (probit QRE), with the variance proportional to the inverse of the precision parameter, $\lambda$. Alternatively, if the perturbations have a double exponential (extreme value) distribution, then the distribution of the difference is logistic, with

$$
p=F(\lambda \Delta(p))=\frac{1}{1+\exp (-\lambda \Delta(p))},
$$

which goes from 0 to 1 as the payoff difference goes from $-\infty$ to $\infty$. The logit probability expression on the right-hand side of (1.4) is a ratio of exponential functions of expected payoffs:

$$
\begin{aligned}
p=\frac{1}{1+\exp (-\lambda \Delta(p))} & =\frac{1}{1+\exp \left(-\lambda \pi_{1}(p)+\lambda \pi_{2}(p)\right)} \\
& =\frac{\exp \left(\lambda \pi_{1}(p)\right)}{\exp \left(\lambda \pi_{1}(p)\right)+\exp \left(\lambda \pi_{2}(p)\right)},
\end{aligned}
$$

where the denominator ensures that the probabilities sum to 1 . With more than two possible actions, the denominator would be a sum of exponential functions of the various expected payoffs. As the precision $\lambda$ goes to 0 , each of the exponential expressions converges to $e^{0}=1$, so the choice probabilities converge to $1 / 2$ (or to $1 / n$ when there are $n$ alternative actions). Conversely, as $\lambda$ goes to infinity, payoff effects dominate noise, and the choice probability for the action with the higher payoff goes to 1 , as can be seen by examining the right-hand side of (1.4), depending on the sign of the expected payoff difference. Finally, if there is a continuum of possible actions, for example, a continuous range of prices or efforts, then the denominator of the logit expression would be an integral over exponentials of expected payoffs (details to follow in a subsequent section).

The endogenous choice probability, $p$, appears on both sides of the equilibrium condition (1.5), which must be solved to determine the equilibrium. Closed-form solutions to this nonlinear equation are generally not available, but numerical methods are straightforward. The game structure determines the expected payoff difference function, and with this, the far right-hand term in (1.5) could be calculated in a spreadsheet, with successive rows for each incremental increase in $p$. The QRE (or equilibria) would be found in rows with a zero difference between the value of $p$ in one column and the quantal response in the other (a finer grid can be used if a more precise calculation is needed). Nonlinear minimization routines for standard programs, such as Matlab or R, can be used to compute numerical solutions for complex games with asymmetries or multiple decisions, and hence, with the entire profile of quantal response choice probabilities to be determined simultaneously. ${ }^{9}$ 
Since the distribution function is increasing, it will have an inverse with the property that $F^{-1}(F(x))=x$. It is straightforward to invert both sides of (1.3), and divide by $\lambda$, to separate the equilibrium condition into terms pertaining to noise on the left and payoffs on the right:

$$
F^{-1}(p) / \lambda=\Delta(p)
$$

This decomposition, with both sides viewed as functions of $p$, can be represented graphically in the top panel of Figure 1.2, where the independent variable, $p$, is on the vertical axis, and expected payoff differences are on the horizontal axis. ${ }^{10}$ The expected payoffs (dashed line) are negative for all values of $p$, as would be the case where decision $D 1$ is dominated and yields lower payoffs regardless of the probability $p$ that the other player chooses $D 1$. The QRE is located at the intersection of the dashed-line expected payoff function and the S-shaped distribution line, which has been graphed so that $F(0)=1 / 2$ as required by symmetry.

The payoff difference line in each panel of Figure 1.2 is derived from a prisoner's dilemma game used by Andreoni and Miller (1993), shown in Figure 1.3. For that game, mutual cooperation yields a payoff of 7 for each, whereas a unilateral defector obtains 12 , so the payoff difference is -5 . This difference determines the intercept of the dashed line with the

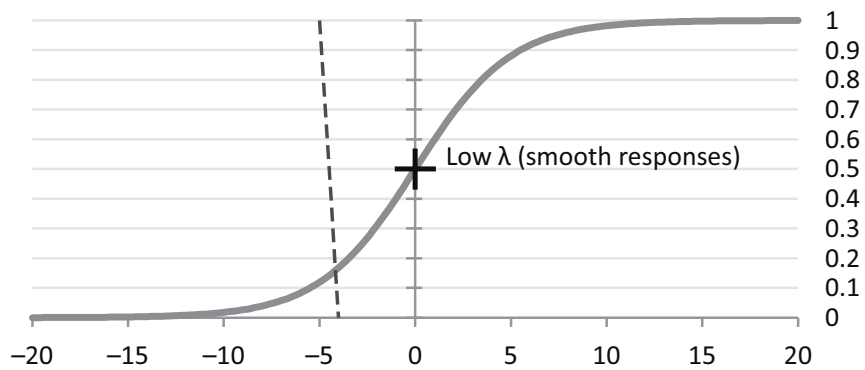

Expected payoff difference for choosing decision $D 1$

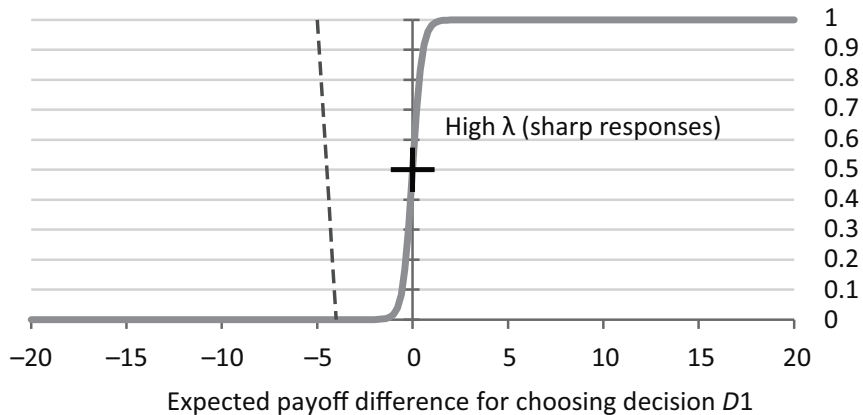

Probability of

decision $D 1$

$\lambda$ scaled distribution

- - - Ex. payoff difference

Probability of

decision $D 1$

$\lambda$ scaled distribution

--- Ex. payoff difference

Notes: The curved cumulative distributions represent a logistic function for $\lambda=0.4$ (relatively high noise) in the top panel, and for $\lambda=4$ (relatively high precision) in the bottom panel. The dashed line to the left of the 0 point shows that expected payoff differences are negative for all values of $p$, which indicates that $D 1$ is a dominated strategy. The intersections determine the QRE, of $p \approx 0.15$ in the top panel and $p \approx 0$ in the bottom panel.

Figure 1.2 QRE for a binary choice game with a dominated strategy, D1 


\begin{tabular}{|c|c|c|c|}
\hline & \multicolumn{2}{|c|}{ Column player } \\
\hline & & Cooperate $(D 1)$ & Defect (D2) \\
\hline \multirow{2}{*}{ Row player } & Cooperate $(D 1)$ & 7,7 & $0, \quad 12$ \\
\hline & Defect (D2) & 12,0 & 4,4 \\
\hline
\end{tabular}

\section{Figure 1.3 A prisoner's dilemma}

top of Figure 1.2. Conversely, when the other person defects, cooperation yields 0 as compared with 4 for mutual defection, so the horizontal intercept of the dashed line in Figure 1.2 is -4 . The dashed expected payoff line connects these two intercepts.

The QRE for the game shown in the top panel of Figure 1.2 involves a positive (but low) probability associated with the dominated decision. An increase in the precision, $\lambda$, transforms the inverse distribution into a shape that looks more like a sharp step function in the bottom panel, with near-zero choice probabilities on the left-hand side where expected payoff differences are negative, and near-unitary choice probabilities on the right-hand side where expected payoff differences are positive. In the limit as precision goes to infinity, the QRE intersection converges to a zero probability associated with the dominated decision, for example, defect in a prisoner's dilemma. The inverse distribution lines in Figure 1.2 were graphed for a logistic distribution with $\lambda=0.4$ in the top panel and with $\lambda=4$ for the bottom panel. The message to take away is that QRE transforms sharp best responses into smoothed better responses, where all actions are chosen with positive probability.

Notice that the payoff structure of the game determines the shape of the dashed payoff difference line in Figure 1.2, and the error structure determines the scaled distribution function. The scaled distribution function has a general shape that depends on the associated precision, but the symmetry properties derived from the i.i.d. error structure will persist. In contrast, various games to be considered will have payoff difference lines with different shapes. For example, a vertical line corresponds to a situation in which the payoff loss from choosing $D 1$ (for example, cooperate) is the same whether or not the other player cooperates or not. The payoff difference line could have a positive slope for some games in which cooperation increases the incentives for others to cooperate. The sections that follow clarify the relationship between the various shapes and slopes of the expected payoff difference lines and the associated QREs.

\section{SYMMETRIC TWO-PERSON GAMES}

The most widely discussed paradigm in game theory is a prisoner's dilemma in which each player has a unilateral incentive to defect (D2), when $c>a$ and $d>b$ for the game in Figure 1.4. The experiment provides an opportunity for learning and possible convergence to equilibrium with multiple rounds of play and random matching between rounds to minimize repeated game effects, for example, the Andreoni and Miller (1993) strangers treatment. Figure 1.2 shows that QRE can explain any cooperation rate for a prisoner's dilemma, as long as it is less than 0.5 . So, in this example, there is some empirical content, but not much, at least without other treatments. 
Column player

\begin{tabular}{cc|cc|cc|}
\multicolumn{1}{c}{} & \multicolumn{2}{c}{$D 1$} & \multicolumn{2}{c}{$D 2$} \\
\cline { 3 - 5 } Row player & $D 1$ & $a$, & $a$ & $b$, & $c$ \\
\cline { 2 - 5 } & $D 2$ & $c$ & $b$ & $d$, & $d$ \\
\cline { 2 - 4 } & & &
\end{tabular}

Figure 1.4 Prisoner's dilemma $(c>a>d>b)$ or battle of sexes $(c>b>a=d)$

Andreoni and Miller (1993) report a prisoner's dilemma with a random matching treatment, using payoffs ( $a=7, b=0, c=12, d=4)$, which resulted in cooperation rates in the 20-25 percent range for the final half of the random pairings. The top panel of Figure 1.5 shows the logit QRE for this prisoner's dilemma using the same precision of 0.4 as was used in the top panel of Figure 1.2. The resulting QRE intersection in the top panel of Figure 1.5 is close to the cooperation rate observed by Cooper et al. (1996). In general, however, there is no a priori reason to expect a precision parameter that provides good predictions for one experiment to also provide good predictions for another experiment with different subjects and different payment protocols. With smooth responses, QRE implies a significant percentage of dominated pro-social $D 1$ choices. ${ }^{11}$ However, for any game with a dominated strategy, the intersection of the expected payoff difference line will involve a choice probability $p$ that is below 0.5 , as indicated by the dark shading along the axis the top panel of Figure 1.5.

Another standard strategic paradigm is a battle-of-sexes game, obtained by reversing the relative magnitudes of $b$ and $d$, which prevents $D 2$ from being a dominant strategy as it would be in a prisoner's dilemma. This would be the case if $c=6, b=2$, and with $a=d=0$, which are the parameters used by Cooper et al. (1989, no-communication treatment) in an experiment using the same random matching protocol as was used in the prisoner's dilemma (Cooper et al. 1996). For the battle-of-sexes game, there is an asymmetric Nash equilibrium in which the row player chooses $D 2$ and the column player chooses $D 1$ (and only earns 2). There is another asymmetric equilibrium, preferred by the column player, in which row chooses $D 1$ and column chooses $D 2$ (and earns 6). With random matching and no communication, players would not be able to coordinate on one of these inequitable equilibria, even if they could agree.

The only symmetric equilibrium for the battle-of-sexes game is one in which each player chooses their preferred decision $D 2$ with probability 0.75 , corresponding to $p=0.25$ for decision $D 1$. This is the probability that makes a player indifferent between the two decisions. (In order to be willing to randomize, the player's expected payoffs for each decision must be equal.) For example, if row chooses $D 1$, there is a 0.75 chance of earning 2 , and if row chooses $D 2$ there is a 0.25 chance of earning 6 , which each yield expected payoffs of $6 / 4$. The bottom panel of Figure 1.5 shows the symmetric QRE analysis for the battle-of-sexes game, using the same precision $(\lambda=0.4)$ as before. The dashed line crosses the vertical center line at $p=0.25$, which is the Nash equilibrium probability $p$ that yields equal expected payoffs (difference of 0 ). The QRE intersection is at approximately $p=0.4$, which is close to the observed frequency of 0.37 reported by Cooper et al. (1989). The negative slope of the expected payoff difference line interacts with the curved distribution function to pull up the QRE intersection from the Nash intersection (of the expected payoff difference line with the vertical line at 0 ) 


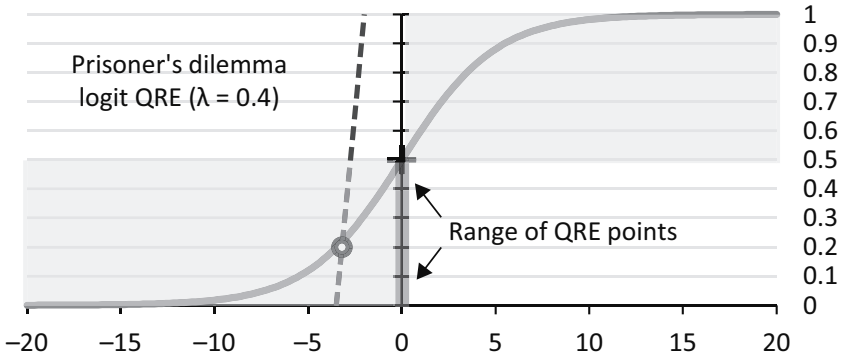

Expected payoff difference for choosing decision $D 1$

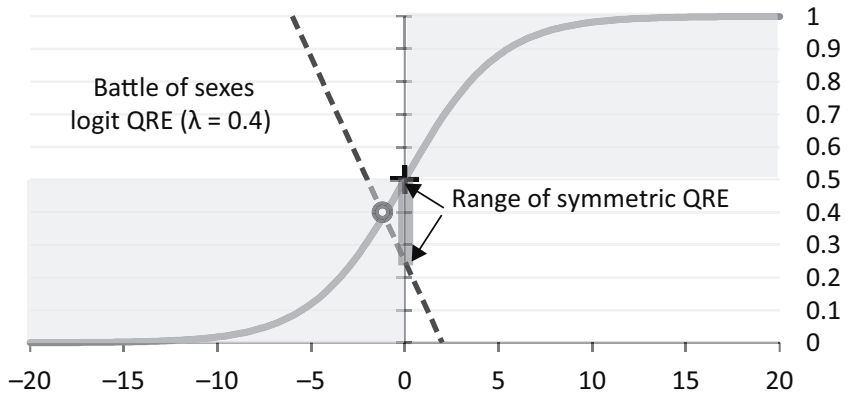

Expected payoff difference for choosing decision $D 1$
Probability of

decision $D 1$

$\lambda$ scaled distribution

- - - Ex. payoff difference

Probability of

decision $D 1$

$\lambda$ scaled distribution

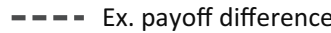

Notes: The scaled distribution lines for both panels are drawn for precisions of 0.4. The dashed-line expected payoff differences are determined by the payoffs used by Cooper et al $(1989,1996)$. In each case (prisoner's dilemma at the top and battle of sexes at the bottom), the symmetric QRE is pulled toward the center relative to the symmetric Nash equilibrium (at $p=0$ in the top panel and at $p=0.25$ in the bottom panel).

Figure 1.5 Logit QRE for a prisoner's dilemma (top) and battle-of-sexes game (bottom)

toward the middle. More noise (lower precision) produces flatter distribution functions, which generates QRE predictions that are closer to 0.5 .

\section{Empirical Content of QRE in the Prisoner's Dilemma and Battle-of-Sexes Games}

Even though the game represented in the top panel of Figure 1.5 has a particular structure (negative expected payoff differences, negative slope), there is a key aspect of the figure that would characterize any binary choice game with i.i.d. payoff perturbations. This is the requirement that the distribution function pass through the point $(0,1 / 2)$, which is owing to neither perturbation being more likely to be larger than the other. That structural characteristic makes it clear that the QRE probability $p$ determined by the intersection point cannot be made arbitrarily large or small by changing the distributions of the i.i.d. perturbations. Adding noise will flatten the distribution, but it will remain monotonic and pass through the midpoint. Therefore, all QRE models with i.i.d. perturbations (logit, probit, and so on, for any precision) will have intersections at probabilities below $1 / 2$ for this game, that is, for this particular expected payoff difference line. For the battle-of-sexes game represented in the bottom panel of Figure 1.5, changes in precision can make the scaled distribution line sharper or flatter, but all possible symmetric QRE intersections are located between the 0.5 midpoint probability and 
the Nash mixed probability of 0.25 (as indicated by the darker shading along the vertical axis). Finally, it is important to note that QRE is not necessarily a parametric theory, although particular parametric (logit or probit) implementations are typically used for purposes of estimation. The empirical content comes from the monotonicity of the $F$ function and the requirement that $F(0)=1 / 2$, which ensures that decisions with higher payoffs are used more frequently.

Graphical representations help to establish an intuitive feel for how the QRE configurations change as precisions and payoffs change. Spreadsheets with fixed references to parameters that can be altered are also useful. To construct the graphical representations used in Figure 1.5, the first step is to calculate the payoff differences. The row player's expected payoff for choosing $D 1$ in the top row of Figure 1.4 is $p a+(1-p) b$, and the expected payoff for choosing $D 2$ is $p c+(1-p) d$, so the expected payoff difference between $D 1$ and $D 2$, denoted by $x$, is: $x=\Delta(p)=p(a-c+d-b)+b-d$. Thus for each value of $x$ on the horizontal axis in Figure 1.5, the vertical coordinate of the payoff difference line would be obtained by solving for $p$ as a function of the $x$ on the horizontal as is: $p=\Delta^{-1}(x)=(x-b+d) /(a-c+d-b)$. The spreadsheet graph is then constructed by creating a column of $x$ values going from -20 to +20 , and then adding a second column with the above formula for the inverse payoff difference: $\Delta^{-1}(x)$, with cell references to payoff parameter specifications. The final step is to add a third column for the distribution function $F(\lambda x)$, with a cell reference to the precision parameter. For a logit equilibrium, the distribution would be determined by the logit distribution in (1.4): $p=1 /(1+\exp (-\lambda x)) .{ }^{12}$ These three columns can then be used to construct a figure with the logit distribution and expected payoff difference lines, where QRE points lie at the intersection(s).

To summarize, quantal response equilibrium with standard assumptions about errors does have empirical content in that it places restrictions on the range of possible choice frequencies. The use of normal or exponential disturbance distributions results in probit or logit QRE, each with a precision parameter that determines the degree of curvature in the better response lines, and the i.i.d. assumption ensures that they will pass through the midpoints $(0,0.5)$ in Figure 1.5. Alternatively, we could use the symmetric structure of the distribution $F(\cdot)$ to derive comparative statics results without making specific parametric distributional assumptions. This is analogous to specifying reduced-form quantal response functions that satisfy basic continuity, responsiveness and monotonicity axioms (Goeree et al. 2005), which ensure that they pass through the center $(0,0.5)$ points. The general structure of these qualitative predictions depends on whether the expected payoff difference line is negatively sloped, as in the bottom of Figure 1.5, or positively sloped, as would be the case where cooperation by one player enhances the other's incentives to cooperate. As shown in the following sections, sometimes QRE exhibits a pull to center, in which probabilities are pulled away from Nash predictions toward probabilities of 0.5 . In other games, QRE exhibits a pull to extremes, in that basins of attraction tend to confer stability properties on QRE that have relatively high or low probabilities compared with a Nash mixed-strategy prediction.

\section{SYMMETRIC $N$-PERSON BINARY DECISION GAMES: APPLICATION TO THE VOLUNTEER'S DILEMMA}

The methods used in the previous sections can be applied to symmetric $N$-person games with a binary choice, for example, contribute or not, choose high effort or not, and so on. Here we 
focus on symmetric equilibria, so that beliefs about the other players can be represented by a probability, $p$, which is used to determine a player's expected payoffs for each of the two decisions. The difference in expected payoffs, denoted by $\Delta(p)=\pi_{1}(p)-\pi_{2}(p)$, determines the QRE when it crosses the scaled inverse distribution of the error difference, as in equation (1.6). The added complexity here is that payoff functions (and their differences) depend on the number of other players, $N-1$, as is the case for the volunteer's dilemma game to be discussed next.

Consider a situation in which it only takes one volunteer to provide a public good for everyone, for example, one person who is willing to attempt a risky rescue or one legislator who is willing to 'take the heat' and propose a pay raise for all. There are $N$ players, and each person's payoff is an amount $V$ if at least one person volunteers (decision $D 1$ ), minus a cost $C<V$ of volunteering for anyone who does so. If nobody volunteers, then all earn a low payoff of 0 . Since volunteering is costly, $C>0$, each person would prefer that someone else volunteers. Alternatively, if nobody else is going to volunteer, then a player would prefer to volunteer since the benefit of $V$ exceeds the individual cost of volunteering: $V>C$. Note that the expected payoff from volunteering is a constant, $\pi_{1}=V-C$, since there is at least one volunteer in this case. The expected payoff from not volunteering (in a symmetric equilibrium with a volunteer probability $p$ ) is the value $V$ times the probability that at least one of the others volunteers: $\pi_{2}=V\left[1-(1-p)^{N-1}\right]$. The difference between volunteer and no-volunteer payoffs is a function of the number of other players: $\triangle(p)=\pi_{1}-\pi_{2}=V(1-p)^{N-1}-$ $C$, which is linear when $N=2$ as shown by the straight, negatively shaped dashed line in Figure 1.6, using parameters $V=0.8$ and $C=0.2$. For higher numbers of players, the payoff differences are reduced as the dashed/dotted lines become increasingly curved.

In a mixed-strategy Nash equilibrium, $p *$, the expected payoffs for volunteering and not volunteering must be equal in order for players to be willing to randomize. That is, the following equation must be satisfied:

$$
C=V\left(1-p^{*}\right)^{N-1}
$$

Therefore, the Nash equilibria are indicated by the dark diamonds in Figure 1.6 on the vertical line above a payoff difference of 0 . This vertical line, of course, corresponds to a 'sharp' distribution function with no dispersion, which illustrates the connection between Nash equilibria and a limit of quantal response equilibria. With a finite precision, $\lambda$, the $S$ shaped distribution line shows some curvature as it passes through the midpoint $(p=0.5$ with a zero payoff difference), as required by the i.i.d. assumption on the payoff disturbances (for example, logit and probit). The intersections with the payoff difference lines at the small circles are the QRE for various group sizes. Note that the QRE prediction for $N=2$ is below the Nash prediction of 0.75 , whereas the QRE predictions are above Nash predictions for the larger groups. This pull-to-center effect would be observed for any parameterization that satisfies the i.i.d. assumption.

It is apparent from Figure 1.6 that as $N$ goes to infinity, the Nash equilibrium volunteer probability goes to zero (as the diamond intersections on the zero-payoff-difference line get lower). Having more players tends to work in the other direction, so the net effect of increasing group size on the chances of getting a volunteer is unclear. This Nash volunteer probability goes to zero so quickly that the probability of getting a no-volunteer outcome is increasing in the number of players. That is, the Nash prediction is that a no-volunteer outcome is 


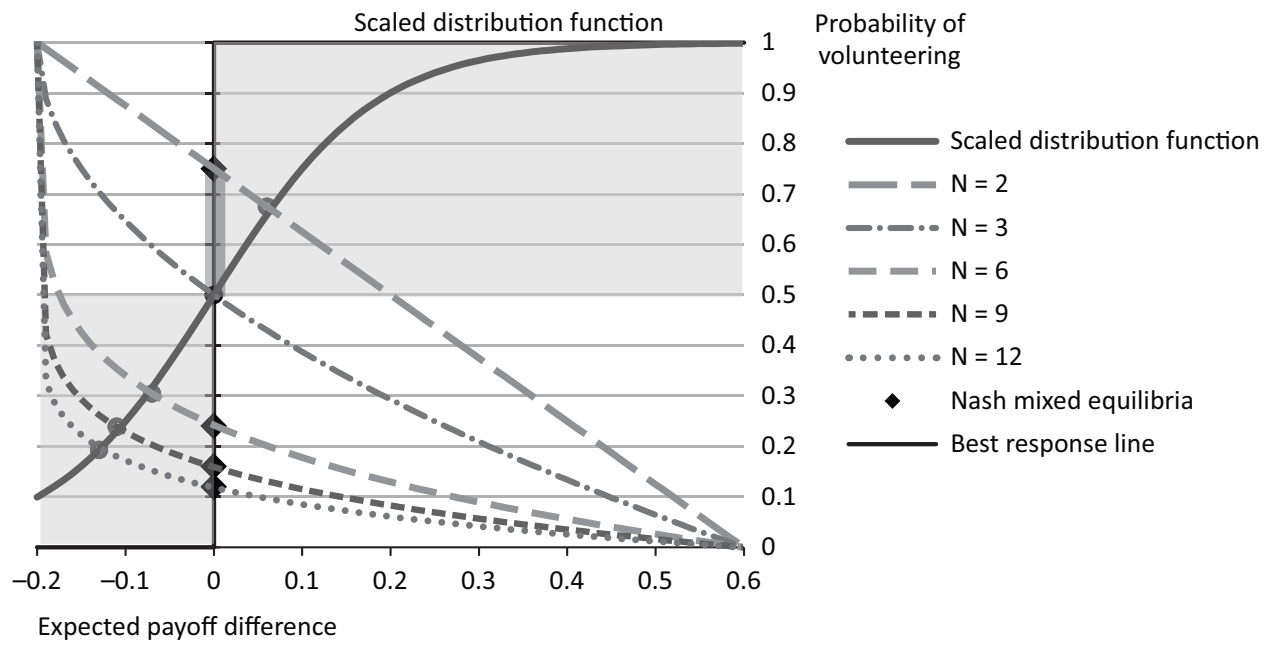

Notes: The curved distribution line is drawn for the logit precision estimated by Goeree et al. (2017), and the expected payoff difference lines are for the parameters used in that paper. The intersections (open circles) are the QRE, which are pulled towards the center relative to the Nash equilibria (diamonds) at the intersections of the expected payoff difference lines and the vertical (sharp) best response line. So the QRE volunteer rate is below the Nash prediction of 0.75 for $N=2$ (as shown by the darker shading along the vertical axis), and it is above the lower Nash predictions for higher numbers of players. As the number of players goes to infinity, it is apparent from the lower-left part of the figure that the QRE probability will not go below 0.1. This lower bound (for a fixed logit precision) will cause the probability of getting no volunteers at all to go to zero, which is consistent with intuition and experimental evidence, but inconsistent with Nash predictions derived from equilibrium volunteer rates that do go to zero as $N$ becomes large.

Figure 1.6 QRE for a volunteer's dilemma with various numbers of players

more likely in a large group. ${ }^{13}$ This unintuitive prediction was not observed in a laboratory experiment in which no-volunteer outcomes diminished steadily as the group size increased from 3 to 12 (Goeree et al. 2017). In contrast, for any given precision $\lambda$, the QRE volunteer rate is bounded away from zero (at a level of about 0.1 where the curved distribution line reaches the payoff difference of -0.2 on the left-hand side of Figure 1.6). This lower bound on the volunteer rate ensures that the QRE probability of getting at least one volunteer goes to 1 for large numbers of players, or equivalently, the probability of a no-volunteer outcome goes to zero. The intuition is that there is always some residual noise, even with large groups, and this noise tends to increase the chances of getting at least one volunteer. Similarly, the incorporation of noise in equilibrium models improves predictions in other experiments in which only a single decision is needed to generate a specific outcome, for example, voting to acquit on a jury subject to unanimity (see the discussion and references in Goeree et al. 2016, ch. 7).

\section{Empirical Content of QRE in the Volunteer's Dilemma}

For each particular group size, it is apparent from Figure 1.6 that the QRE predictions lie between the Nash prediction and 0.5, a pull-to-center effect that was present for all four group-size treatments with predictions that differed from 0.5 . Therefore, if the data average 
were outside of this range between Nash and 0.5, it would be impossible to find a precision that generates a perfect fit, even for a single treatment. For a group size of 2, for example, the straight dashed expected payoff difference line passes through the northeast shaded rectangle over a range of $p$ values between 0.75 and 0.5 (the dark shading along the vertical axis). The empirical content for the volunteer's dilemma game is sharpened by the presence of data for a wide range of treatments that were used to estimate a single precision parameter. Notice that these comparative statics predictions do not depend on any particular parametric form (logit, probit, and so on) of the quantal response function $F$, since any cumulative distribution resulting from i.i.d. disturbances would be increasing and would pass through the $(0,1 / 2)$ point in Figure 1.6. Moreover, the arguments just presented imply that the probability of no-volunteer outcomes is decreasing for large groups, not increasing as implied by the Nash equilibrium. That is, there is no finite value of the precision parameter that would fit data that happened to conform to the Nash prediction pattern of a no-volunteer rate that is increasing in $N$.

\section{COORDINATION GAMES AND MULTIPLE, PARETO-RANKED EQUILIBRIA}

A coordination game is one with multiple Nash equilibria, so that players have to solve the problem of coordinating on a preferred equilibrium. For example, suppose that the incentive to defect unilaterally from the cooperative outcome in a prisoner's dilemma (the $c$ payoff in Figure 1.4) is reduced to the extent that that there is no longer an incentive to defect, that is, $a>c$. In this example, the original cooperation outcome would constitute a second Nash equilibrium that is preferred by both players to the mutual defection equilibrium. Coordination games have fascinated economists and game theorists, owing to the possibility that players may fail to coordinate on the Pareto-preferred equilibrium. This possibility is especially credible in games that require coordination among many players, for example, if the failure of some to coordinate may increase the attractiveness of the 'bad' equilibrium for others. Malthus, for example, worried that a whole economy might become mired in a general glut that could be hard to escape. One example of this situation is the weakest-link game, for which the payoff for each player is a function of the minimum of all players' efforts. One player's actions can weigh heavily on the others' payoffs in this weakest-link, or minimum-effort, game. Laboratory experiments confirm that coordination on good equilibria is especially difficult with larger numbers of players in a weakest link game (Van Huyck et al. 1990; Goeree and Holt 2003a, 2005a).

Figure 1.7 shows the payoffs for a two-person weakest link game for which decision $D 1$ corresponds to a high effort with a cost of $2 C$, and decision $D 2$ corresponds to a low effort with a cost of $C$. When both subjects choose low effort, the minimum is 1 unit, and they each earn $V-C$, as shown in the lower right-hand box. If the row player unilaterally raises effort to the high level, the effort cost goes up, but the minimum stays low, so the lower right-hand box is a Nash equilibrium. Alternatively, when both choose a high effort, the minimum is high and they each earn $2 V-2 C$, as shown in the upper left-hand box of Figure 1.7. Starting at this good outcome, a unilateral effort reduction by the row player would lower both the minimum effort and the cost, that is, the row player's payoff would fall from $2 V-2 C$ to $V-C$. Thus a unilateral effort reduction from the high-effort equilibrium is not profitable. The dilemma 


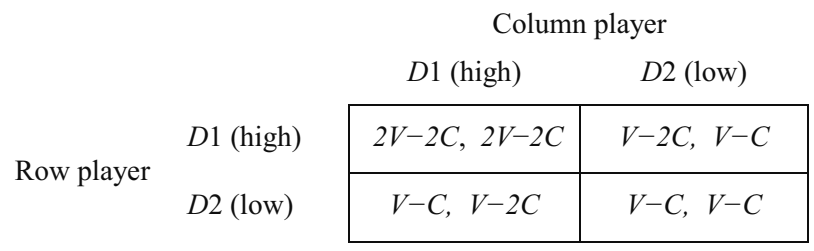

Figure 1.7 Weakest link (minimum effort) coordination game $(V>C>0)$

is that low effort for both is also Nash equilibrium. The payoffs are twice as large for each if they both choose high effort, but there is no risk in choosing low effort. In addition to the two pure-strategy Nash equilibria at high- or low-effort levels, there is also a mixed-strategy Nash equilibrium at an intermediate probability of high effort, $p$, which equates the expected payoffs of the two effort choices.

Figure 1.8 shows the previously discussed decomposition of the dashed expected payoff difference line and the curved ( $\lambda$-scaled) distribution line, the intersections of which determine QREs (circles in the figure). Note that the expected payoff difference line for a two-player coordination game has a positive slope, which reflects the intuition that a higher probability that the other player chooses high effort raises the payoff difference for a choice of high effort. The game being represented has an effort cost of $C=0.4$ and a value of $V=1$. The dashed expected payoff difference line starts at -0.4 on the horizontal axis because when the other chooses high effort with probability 0 (bottom of Figure 1.8), an extra unit of effort has no effect on the minimum; it just reduces one's payoff by 0.4 . Conversely, when the other player chooses high effort, $p=1$, a switch to high effort raises the minimum effort and the cost, for a net gain of $V-C=1-0.4=0.6$, as shown by the intersection of the dashed line with the top of Figure 1.8.

As indicated from the previous analysis of equation (1.4), the QREs correspond to intersections of the curved distribution line and the dashed expected payoff difference line. In Figure 1.8, the circle at the top is for the outcome where both players choose high effort with a probability that is close to 1 , and the circle at the bottom is for the other equilibrium where the probability of high effort is close to 0 . The middle QRE circle in Figure 1.8 is near the Nash mixed equilibrium, which is at the intersection of the expected payoff difference line and the vertical line at a payoff difference of 0 that makes a player indifferent and willing to randomize. This illustrates the general tendency for QRE points to be near Nash equilibria when precision is relatively high (Goeree et al. 2016, ch. 1, and references therein).

The $\lambda$-scaled distribution line in Figure 1.8 is drawn with a specified level of the disturbance precision $\lambda$. A lower precision would result in a flatter line, which would move the two extreme QRE circle predictions inward, away from the Nash equilibria where the probability of high effort is either 0 or 1 . A sufficiently low precision could cause the distribution line to flatten out so that there is only one intersection with the payoff difference line, at a probability above 0.5 . That is, the QRE with a relatively high probability of high effort may vanish for sufficiently low precision levels. This is consistent with intuition that coordination on a high-effort outcome is difficult in the presence of noise that increases the risk of choosing high effort.

There is a simple intuition suggesting that the middle QRE point in Figure 1.8 (at a higheffort probability of about 0.37 ) could be unstable. For example, beginning at a probability 


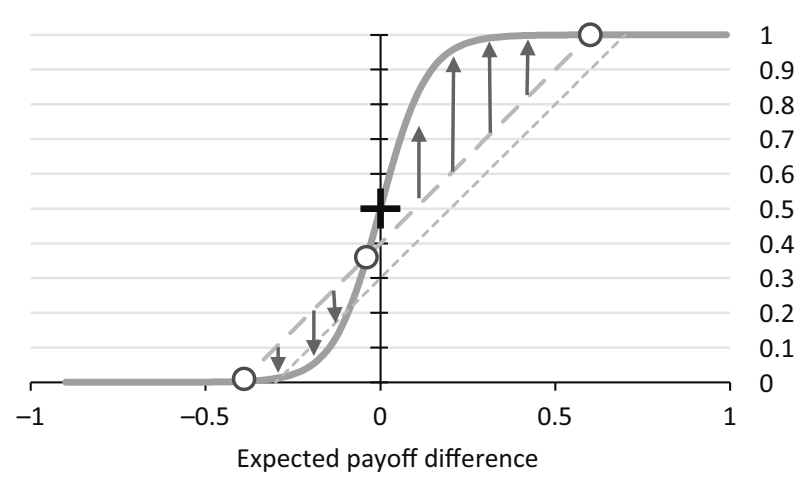

Probability of

high effort

0.8

0.7

Lambda scaled distribution

- - Payoff difference $\mathrm{N}=2$

Payoff difference $\mathrm{N}=2$, reduced effort cost

Notes: The expected payoff difference (for choosing high effort instead of low effort) is shown on the horizontal axis, and the probability $p$ that the other player chooses high effort is shown in the vertical axis. The straight dashed line shows the expected payoff difference for high effort as a function of $p$ for the case of $N=2$ players. The slope of this expected difference line is positive, since a higher probability that the other chooses high effort raises the expected payoff gain of switching to high effort. The QRE points are at the intersections of the payoff difference line and the curved distribution line. Two of these equilibria (the circles near the top or bottom boundaries) represent the high-effort and low-effort QRE outcomes. The thin dashed line shows the rightward shift in the expected payoff difference resulting from a reduction in the effort cost.

\section{Figure 1.8 A two-player minimum-effort coordination game with $V=1$ and $C=0.4$}

of 0.5 in the center of Figure 1.8, move to the dashed line to the right to determine a positive payoff difference. Then move up vertically to the curved probabilistic response for this positive payoff difference, which is much higher than 0.5 . This intuition suggests that if choice proportions start near 0.5 , the incentives to choose high effort will cause quantal responses to raise the proportion of high effort decisions, as suggested by the upward arrows shown in that region. The converse reasoning applies for probabilities that are below those of the middle QRE circle in Figure 1.8, that is, quantal responses tend to pull probabilities down in this region. In effect, the middle QRE determines the basins of attraction for the extreme, high-effort or low-effort QRE. In this case with two players, the high-effort QRE has a larger basin of attraction. The instability of the middle QRE point is consistent with the unintuitive comparative statics for that point. For example, a reduction in the cost of effort might be expected to increase the probability of a high-effort decision. However, a decrease in $C$ raises the expected payoff differences and shifts the payoff difference line to the right in Figure 1.8 (the thin dashed line). This shift causes the middle QRE intersection to be at a lower probability of high effort. What is intuitive is that a reduction in the cost of effort, by lowering the middle QRE point, will increase the basin of attraction for the high-effort QRE point.

With $N$ players, a high-effort decision results a sure cost of $2 C$. A high common-effort outcome only results if all others choose high effort, which happens with probability $p^{N-1}$. A low effort yields a sure payoff of $V-C$. In this instance, the expected payoff difference function is $\Delta(p)=V p^{N-1}-C$, since adding a unit of effort may possibly raise the value received, but surely raises the cost. This payoff difference function for $N=3$ will be quadratic, shown as the curved dot-dash line in Figure 1.9. For these parameters with $C=0.4$ and $V-C=0.6$, the expected payoff difference line still passes through the points $(-0.4,0)$ and $(0.6,1)$, as was the case for two players. However, the expected payoff line is located to the left for intermediate probabilities, since the probability that the minimum of 


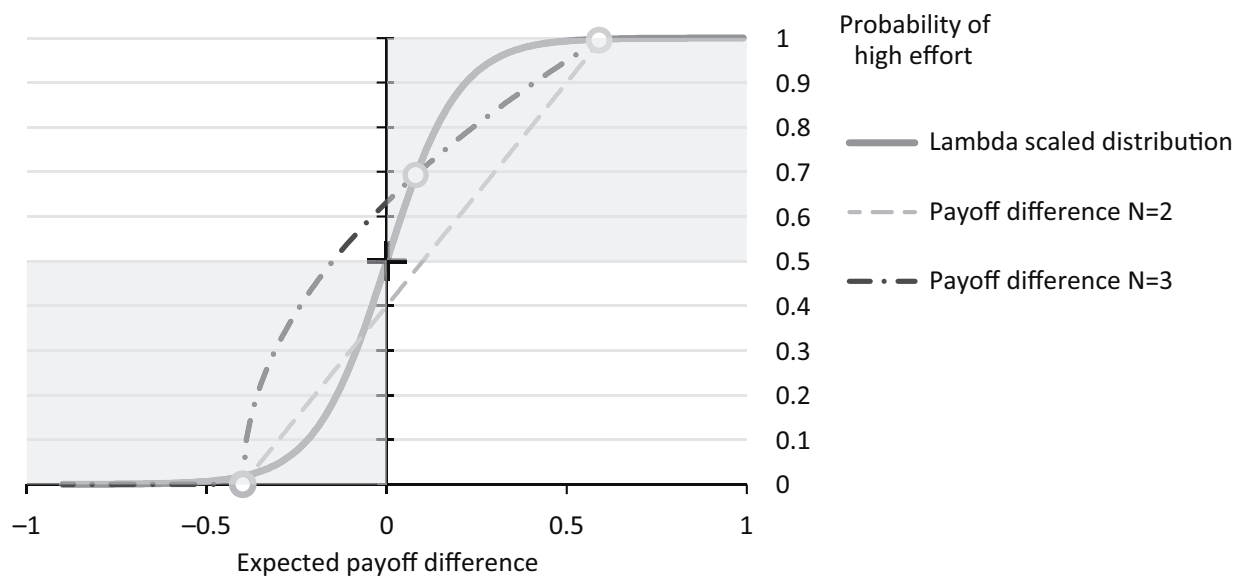

Notes: With three players, the expected payoff difference depends on the probability that both other players choose high effort, which is $p^{2}$. This nonlinearity causes the expected payoff difference line to have curvature, as shown by the dot-dash line. This line has shifted up and to the left relative to the straight dashed line for $N=2$, although the expected payoff intercepts at the top and bottom of the figure are the same. As before, the QRE points are at intersections between the expected payoff difference line and the curved distribution line. There is one QRE with relatively low effort (circle intersection near the bottom), another with relatively high effort (circle near the top), and an unstable QRE with an intermediate probability. Notice that the quantal responses are below the payoff differences anywhere to the left of the unstable QRE point, which tends to pull down the probability of high effort. Thus the increase in the number of players has increased the region of attraction for the low-effort QRE.

Figure 1.9 A three-player weakest link coordination game with $V=1$ and $C=0.4$

others' efforts is high is decreasing in the number of other players. As before, there are three QREs (circles in Figure 1.9), one near the upper boundary, one near the lower boundary and an intermediate equilibrium that is unstable and that delimits the basins of attraction. An increase in the number of players would increase the basin of attraction for the low-effort equilibrium, as would be suggested by simple intuition about the increased difficulty of coordination for a larger number of players.

\section{Empirical Content of QRE in Coordination Games}

Recall that the curved line in Figures 1.8 and 1.9 is the $\lambda$-scaled distribution of the difference in payoff disturbances. This distribution function must be upward sloping and pass through the cross at the center of each figure. This point at $(0,0.5)$ is where the cumulative probability is 0.5 , reflecting the i.i.d. assumption that neither of the disturbances is more likely to be larger than the other. As precision becomes smaller, the scaled distribution line becomes flatter, but since it is non-decreasing, it can only pass through the points in the light shaded areas. Thus the distribution line can never intersect with the payoff difference line in the region to the southeast (down and to the right) of the cross at the center of Figure 1.9, or to the northwest (up and to the left). With two players, the standard i.i.d. assumption about disturbances precludes any QRE in this region, at probabilities between about 0.4 and 0.5 as shown where the light thin dashed line passes in the open between the two shaded rectangles in Figure 1.9. Similarly a non-decreasing cumulative distribution cannot intersect the payoff difference for the case of $N=3$ at any probability between 0.5 and about 0.65 in the three-person coordination game. 
To summarize, when the expected payoff difference line is positively sloped, as in coordination games, for plausible values of lambda, the standard i.i.d. assumptions preclude QRE in probability ranges close to .5 (in one direction or the other), just as the pull-to-center QRE tendencies with negatively sloped expected difference lines tend to rule out QREs that are located in regions closer to the extremes of 0 or 1 . Finally, note that coordination on high efforts is more difficult with more noise, more players attempting to coordinate and a higher cost of effort.

\section{GAMES WITH ASYMMETRIC EQUILIBRIA}

In an asymmetric equilibrium, each player's choice probability will be determined by the player's beliefs about the other's decision. In a Nash equilibrium, the choice probabilities for each player will be best responses to beliefs about the other's decision. The associated best response lines will have sharp edges, with Nash points at the intersections. In a QRE, each player's choice probability will be a noisy response, which can be represented by a curved quantal response line for each player. With two players and two decisions, the QRE probabilities are located at the intersection of the players' quantal responses. As precision decreases, curvature increases, which can move the QRE point away from Nash in the presence of payoff asymmetries.

Figure 1.10 represents a matching pennies game, in which the row player prefers a match on heads or tails, and the column player prefers a mismatch. This game is highly asymmetric because the row player prefers matching on heads to matching on tails. Payoffs would be symmetric if the 10 were reduced to 2 , in which case the Nash equilibrium would be for each player to randomize with probabilities of $1 / 2$ for each decision. However, if the row player's top left payoff is 10 as in Figure 1.10, the row player must still randomize by choosing top with probability of only $1 / 2$ in order to keep the column player indifferent and willing to randomize. This stark Nash equilibrium prediction of no own-payoff effect is not supported by laboratory experiments with random matching. Instead, row players tend to choose top more often than bottom (Ochs 1995; Goeree et al. 2003).

Consider the best response for the column player, shown as a dashed line in the left panel of Figure 1.11. The symmetry of the column player's payoffs implies that it is a best response to switch from left to right as the row player's probability of top crosses $1 / 2$, as indicated by the dashed line in the left-hand panel of Figure 1.11. A quantal response function would be smoother, represented by a curved line that would lie somewhere in the lower shaded area to the left of $1 / 2$ and in the upper shaded area to the right.

\begin{tabular}{|c|c|c|c|}
\hline & \multicolumn{2}{|c|}{ Column player } \\
\hline & & Left (heads) & Right (tails) \\
\hline \multirow{2}{*}{ Row player } & Top (heads) & 101 & 12 \\
\hline & Bottom (tails) & 12 & 21 \\
\hline
\end{tabular}

Figure 1.10 Asymmetric matching pennies game 

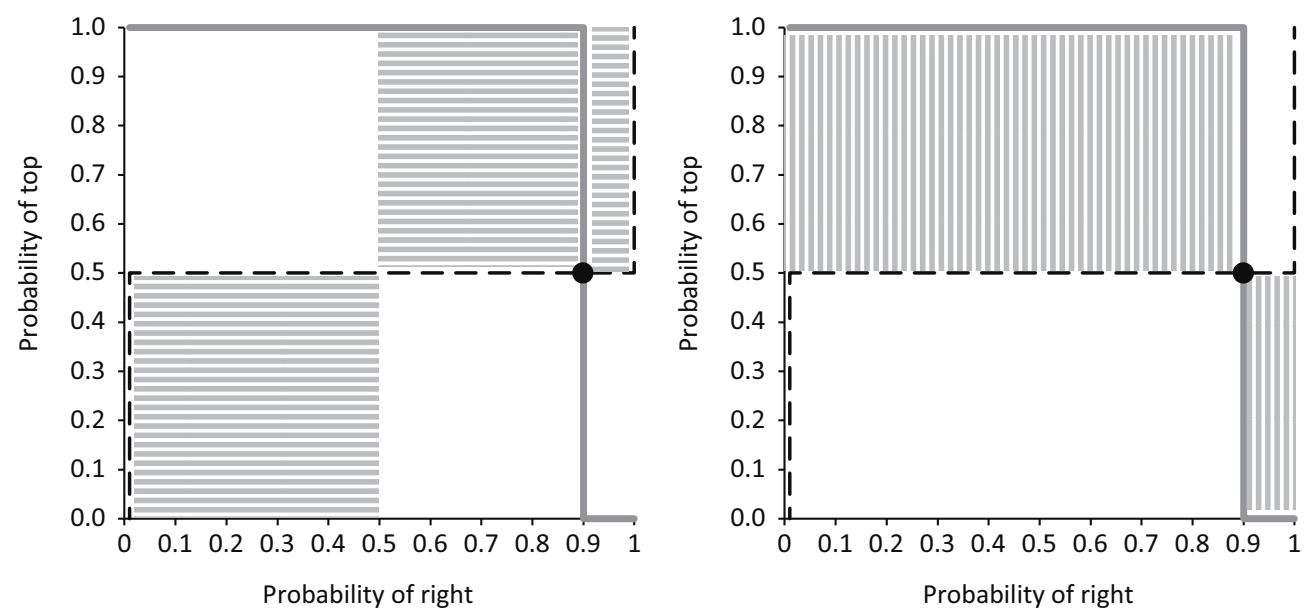

Notes: As the precision decreases, the sharp dashed best response for the column player will flatten out into quantal responses, but it will stay in the horizontal shaded regions in the left-hand pane. Similarly, the vertical shaded regions in the right-hand panel show the possible locations of the quantal responses for the row player.

Figure 1.11 Best response lines for a matching pennies game: column player (dashed lines) and row player (solid lines), with shading for possible locations of quantal responses

Conversely, in order to make the row player indifferent in this asymmetric game, the column player must choose right with probability 0.9 , as shown by the row player's vertical solid best response line in the right-hand panel of Figure 1.11. For probabilities of right below 0.9, the row player's best response is to choose top for sure, as shown by the best response line in the right-hand panel of Figure 1.11 that comes across the top of the panel and then drops to 0 when the probability of right is equal to 0.9 , at which point the row player's expected payoffs for each decision are equal. With some noise, the best response line is replaced by a smoothed line that starts somewhere the upper-left shaded area and crosses to the lower-right shaded area.

If there is an i.i.d. payoff disturbance associated with the expected payoff for each decision, then the quantal responses can be represented by scaled distribution functions $F$ of the expected payoff difference, $\Delta(p)$ that depends on beliefs about the other player's decision, that is, the row player's beliefs about $\operatorname{Pr}$ (Right) and column's beliefs about $\operatorname{Pr}(\operatorname{Top})$. These quantal responses are shown in equations (1.8) and (1.9), with the logit specification shown after the second equal sign:

$$
\begin{gathered}
p_{\text {Top }}=F\left(\lambda \Delta_{\text {Row }}\left(p_{\text {Right }}\right)=\frac{\exp \left(\lambda \pi_{\text {Top }}\left(p_{\text {Right }}\right)\right)}{\exp \left(\lambda \pi_{\text {Top }}\left(p_{\text {Right }}\right)\right)+\exp \left(\lambda \pi_{\text {Bottom }}\left(p_{\text {Right }}\right)\right)},\right. \\
p_{\text {Right }}=F\left(\lambda \Delta_{\text {Column }}\left(p_{\text {Top }}\right)=\frac{\exp \left(\lambda \pi_{\text {Right }}\left(p_{\text {Top }}\right)\right)}{\exp \left(\lambda \pi_{\text {Right }}\left(p_{\text {Top }}\right)\right)+\exp \left(\lambda \pi_{\text {Left }}\left(p_{\text {Top }}\right)\right)},\right.
\end{gathered}
$$




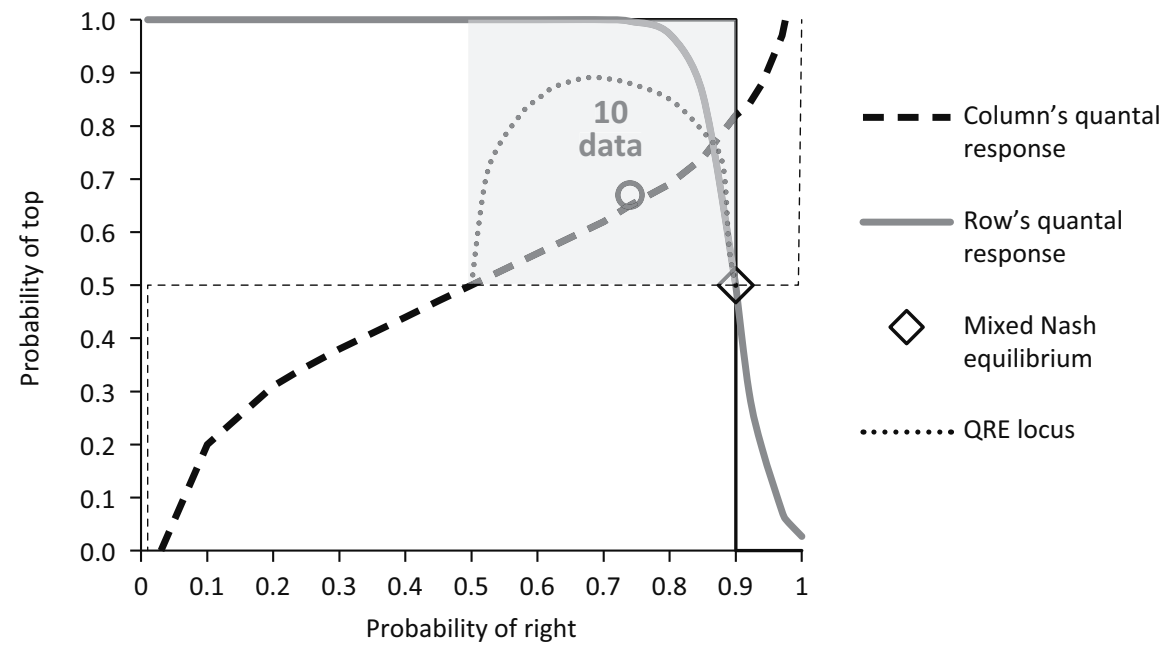

Notes: The curved quantal response lines will intersect in the shaded area, which is the intersection of the shaded sets in Figure 1.11. This set illustrates that QRE does have empirical content in this game when payoff disturbances are i.i.d. The particular curved quantal response lines shown are for a precision that was estimated using data from two different matching pennies games. The intersection of the quantal response lines is above and to the left of the Nash equilibrium (hollow diamond). The hollow dot (marked ' 10 data') shows the average of the proportions of top and right for row and column players, respectively, in an experiment. Thus QRE explains the qualitative nature of the deviations from the Nash prediction. In particular, the row player is responsive to the high payoff of 10 for a top/left outcome, and therefore, the row player chooses top more often. The arc of small dots shows the locus of all QRE points when disturbance precisions for each player are constrained to be equal.

Figure 1.12 Best responses (straight lines) and quantal responses (curved lines) for the column player (dashed lines) and row player (solid lines)

In a QRE, the $p_{\text {Top }}$ and $p_{\text {Right }}$ beliefs on the right sides of (1.8) and (1.9) must match the choice probabilities on the left, so the QRE is obtained by solving these two nonlinear equations. This solution can be represented by an intersection of quantal response lines in Figure 1.12. Those particular curves represent the logit quantal response functions, for the specific value of the precision parameter, $\lambda$, that was estimated from laboratory data of two asymmetric matching pennies games, one shown in Figure 1.10 and a second treatment where the 10 payoff was reduced to 10/9. The data average is represented as the hollow dot ' 10 data' in Figure 1.12. Even though the QRE prediction is not perfect for the 10 treatment, it is qualitatively similar in terms of the observed frequency of top being significantly above the 0.5 Nash prediction, and the observed frequency of right being significantly to the left of the Nash prediction of 0.9. That is, the QRE exhibits the own-payoff effect of the high 10 payoff that is apparent in the data but not implied by a Nash equilibrium.

Finally, the shaded area in Figure 1.12 represents the intersection of the shaded regions of the two panels of Figure 1.11. In order to reach all parts of the shaded regions in Figure 1.12, we would need to have the flexibility to choose one precision for the row player and another for the column player. Since roles in the experiment are randomly assigned, it is better to model the precisions as being the same, which would sharply restrict the shaded region of possible QREs in Figure 1.12. For any specific parametric specification, the locus of QRE points would start at the central point of Figure $1.12(0.5,0.5)$ as precision goes to 0 , rise into 
the shaded region, and would end up at the Nash mixed equilibrium $(0.9,0.5)$ as precision goes to infinity (dotted arc in Figure 1.12). The locus of all logit QRE points with a single precision parameter is a curved line, not an area.

\section{Empirical Content of QRE in Matching Pennies Games}

If payoff disturbances are i.i.d. (for example, logit and probit) then the set of possible QREs for the asymmetric matching pennies game in Figure 1.10 is the shaded set in Figure 1.12. Goeree et al. (2019) generalizes this nonparametric restriction implied by QRE for arbitrary finite games and show that the relative measure of the shaded region is bounded above by $1 / K$ !, where $K$ is the number of available actions in the game for a player. That set is further restricted if the payoff disturbances are identically distributed according to a specified parametric distribution, for example, logit with the same precision, in which case the set of possible QRE is a line not an area. As always, empirical content is further sharpened when multiple treatments with different payoff parameters are used, for example, Holt and Palfrey (2020).

\section{QUANTAL RESPONSE EQUILIBRIUM IN EXTENSIVE FORM GAMES: THE CENTIPEDE GAME}

Many games of interest - for example, signaling games, repeated games and multistage bargaining games - have a timing structure that is not captured in the strategic-form representation. Importantly, subgame perfection and sequential rationality lead to strong restrictions on equilibrium behavior, and are appropriately analyzed as games in extensive form. McKelvey and Palfrey (1998) develop the theoretical framework for QRE in extensive form games, naming it agent quantal response equilibrium (AQRE). The terminology is based on a player's decisions at subsequent decision nodes being modeled as decisions of a noisy agent subject to payoff disturbances at those future nodes. While the formal development of the theory is complicated, it is easy to describe for multistage games of perfect information. Consider, for example, the logit AQRE. Starting from the final stage, the last player chooses stochastically according to the logit quantal response function with precision parameter $\lambda$ at each information set, where the outcomes of the player's possible choices are known quantities, so no expectation over other players' action choices are necessary at this last stage. This, in turn defines the expected payoffs for all available actions at each information set for the next-to-last player. That player then logit quantal responds in the next to last stage. In this manner, the game is solved backwards until the initial stage.

This basic idea extends in a natural way to any extensive-form game with perfect recall. At each information set for each player, a player's continuation expected payoffs (for each available action at that information set) are calculated using the distribution over the terminal payoffs based on the future quantal responses of all players at all information sets following that move. The formal structure is laid out in McKelvey and Palfrey (1998). This backward induction process guarantees that the limits of $\mathrm{AQRE}$, when $\lambda$ goes to infinity, are sequential equilibria of the underlying game, so AQRE imposes a quantal response version of sequential rationality. Since the quantal responses are strictly interior, in any AQRE, every information set is reached with positive probability. Thus, unlike the standard equilibrium analysis of 
extensive-form games, there is no need to arbitrarily assign beliefs to unreached information sets, where Bayes' rule fails to apply. Instead, Bayes' rule applies everywhere in the game, thereby completely avoiding any issues related to belief-based refinements of the Nash equilibrium.

We next illustrate the quantal backward induction method for the four-stage centipede game shown in Figure 1.13. In each stage of this game, the player moving at that stage chooses to either pass the game to the next stage, or to take and receive a payoff that is four times the payoff of the other player. The take decisions are shown as down arrows and the pass decisions are shown as arrows to the right. For example, player 1 decides first at the left node, and a take (down arrow) at that point results in payoffs of 40 for player 1 and 10 for player 2. Notice that if a player chooses pass, the payoff amounts double and are switched. It is as if unequal money amounts are passed in a tray, with the person making the decision deciding to take the higher amount or pass, which doubles the money amounts but offers the other player the chance to take the higher amount. If nobody has taken in the first four stages, the game ends with pass payoffs of $(640,160)$ for players 1 and 2 respectively, as shown on the right-hand side of Figure 1.13. McKelvey and Palfrey (1992) studied behavior in this game and in a six-stage extension (with payoffs in pennies) of $(640,160)$ for a take stage $5,(320,1280)$ for a take in stage 6 , and with the final pass payoffs being $(2560,640)$ for players 1 and 2 respectively.

All Nash equilibria in the centipede game (and there is more than one) involve player 1 taking in the first stage. There is a unique sequential equilibrium where each player takes whenever it is their move. Behavior observed in laboratory experiments is far different. The top part of Table 1.1 displays the empirical relative frequencies at which players were observed to choose take at each stage, $t$, denoted by $q_{t}$. The striking pattern in the data is the strong horizon effect, observed in both treatments: take probabilities start near zero and increase dramatically as the game progresses and the final stage is approached.

Zauner (1999) and McKelvey and Palfrey(1998) used the observed take rates to estimate a quantal response precision parameter using different specifications of the quantal response functions. ${ }^{14}$ In a logit QRE, for example, increases in the payoff differences between take and pass in later stages of the centipede game would result in higher take rates in later stages, essentially moving up along a fixed lambda-scaled cumulative distribution function in Figures

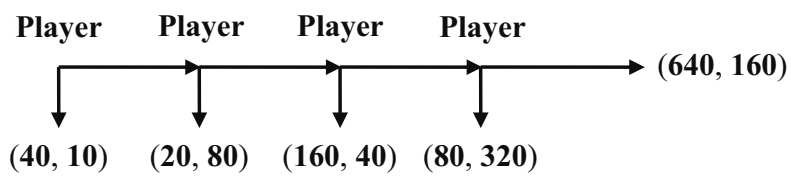

Take rates:

$\begin{array}{llll}0.07 & 0.38 & 0.64 & 0.75\end{array}$

\begin{abstract}
Notes: Player 1 begins at the node on the left by choosing between take (down arrow) and pass (right-pointing arrow), where a take results in payoffs of 40 cents for player 1 and 10 for player 2 . At the second node, player 2 chooses between take (down) and pass (right), and decisions alternate if the game continues. The bottom row shows rates of take decisions observed by McKelvey and Palfrey, which increase steadily from 0.07 in the first stage to 0.75 in the final stage.
\end{abstract}

Figure 1.13 Four-stage centipede game and observed take rates (McKelvey and Palfrey 1992) 
Table 1.1 Take rates in four-and six-stage centipede games: observed (McKelvey and Palfrey 1992) and Luce QRE fitted rates with $\lambda=1.16$

\begin{tabular}{lllllll}
\hline Take rates & $q_{1}$ & $q_{2}$ & $q_{3}$ & $q_{4}$ & $q_{5}$ & $q_{6}$ \\
\hline 4-move observed: & 0.070 & 0.380 & 0.640 & 0.750 & - & - \\
6-move observed: & 0.007 & 0.065 & 0.210 & 0.530 & 0.730 & 0.850 \\
\hline 4-move fitted Luce: & 0.160 & 0.270 & 0.370 & 0.690 & - & - \\
6-move fitted Luce: & 0.050 & 0.100 & 0.160 & 0.270 & 0.370 & 0.690 \\
\hline
\end{tabular}

1.1-1.2 and 1.5-1.6. Recall that the take payoffs double (and switch) in each subsequent stage of the centipede game in Figure 1.13. Since logit choice probabilities are constructed from ratios of exponential functions of expected payoffs, a doubling of all payoffs that doubles all expected payoffs is analogous to doubling the logit precision, as can be seen from the location of the two numbers adjacent to the precision $\lambda$ in equation (1.10).

$$
p=\frac{\exp \left(\lambda 2 \pi_{1}(p)\right)}{\exp \left(\lambda 2 \pi_{1}(p)\right)+\exp \left(\lambda 2 \pi_{2}(p)\right)} \text { (logit with doubled payoffs). }
$$

This observation suggests that behavior should become much more precise in later stages of the centipede game. In contrast, note that the take rate in the final, fourth, stage in Figure 1.13 is only 0.75 . An alternative to the logit form that is sometimes used when payoff scale changes are large is the Luce probabilistic choice rule:

$$
p=\frac{\left(\pi_{1}(p)\right)^{\lambda}}{\left(\pi_{1}(p)\right)^{\lambda}+\left(\pi_{2}(p)\right)^{\lambda}} \text { for } \lambda>0 \text { (Luce power rule), }
$$

where $\lambda=0$ implies extreme noise and choice probabilities of 0 . Notice that this choice function is invariant to uniform changes in payoff scale, for example, doubling. Thus the Luce rule implies that choice probabilities depend on ratios of expected payoffs, not on differences. ${ }^{15}$ Next we illustrate the use of the Luce rule in the context of the centipede game.

An AQRE for the four-move game is a sequence of take probabilities, $\left(q_{1}^{*}, q_{2}^{*}, q_{3}^{*}, q_{4}^{*}\right)$. At stage $j$, where $q_{j}^{*}$ is a quantal response to the expected payoffs from the take decision to the player moving at that stage, given the take rates, $q_{k}^{*}$, for all subsequent stages $k>j$. At stage 2 , the quantal response for the Luce power rule (1.11) is constructed as a ratio of terms, each of which is a payoff raised to the power $\lambda$ :

$q_{2}^{*}=\frac{(80)^{\lambda}}{(80)^{\lambda}+\left[40 q_{3}^{*}+\left(1-q_{3}^{*}\right)\left(320 q_{4}^{*} *+160\left(1-q_{4}^{*}\right)\right)\right]^{\lambda}}($ Luce rule for stage 2$)$.

The numerator in (1.12) is the payoff for player 2 from take, 80, raised to the power $\lambda$, and the denominator is the sum of that term and a second term that represents the expected payoff from passing, also raised to the power $\lambda$. The best fitting precision value for the take-rate data shown in the top part of Table 1.1 is $\lambda=1.16$, generating predicted take probabilities as 


\section{Handbook of experimental game theory}

shown in the bottom part of Table 1.1. Notice that the qualitative horizon effect observed in the top part is clearly captured in the estimates shown in the bottom part.

It is also instructive to look at the entire power Luce QRE correspondence for this 4-stage game, shown in Figure 1.14. Begin on the left with a zero precision (perfect randomness), which causes all take probabilities to be 0.5 . Figure 1.14 shows that convergence to the unique subgame perfect equilibrium, even in games of perfect information, is not necessarily monotone in $\lambda$. Only $q_{4}^{*}$ converges monotonically to 1 , because a pass at node 4 is a strictly dominated strategy. In contrast, $q_{1}^{*}, q_{2}^{*}$, and $q_{3}^{*}$ initially decrease because for low values of $\lambda$ there is sufficient noise in the future play of the game such that passing yields a higher expected payoff, so the associated take rate is below a half. For all strictly positive precision values, however, the lowest take rate is in the first stage (dark thick line) and take rates are higher at each subsequent stage (lighter lines), so each specific value of the precision parameter exhibits the same pattern of increasing take rates from stages 1 to 4 . Therefore, this correspondence shows how QRE captures the horizon effect for a wide range of $\lambda$ values. The vertical dashed line is drawn at the best-fit value of $\lambda$, and the intersections with the curved lines show the resulting best-fit QRE predictions.

\section{Empirical Content of QRE in the Centipede Game}

It is apparent from Figure 1.14 that a perfect fit for a single game would require four observed take rates to lie on the same vertical line, and the likelihood of a perfect fit for both four-stage

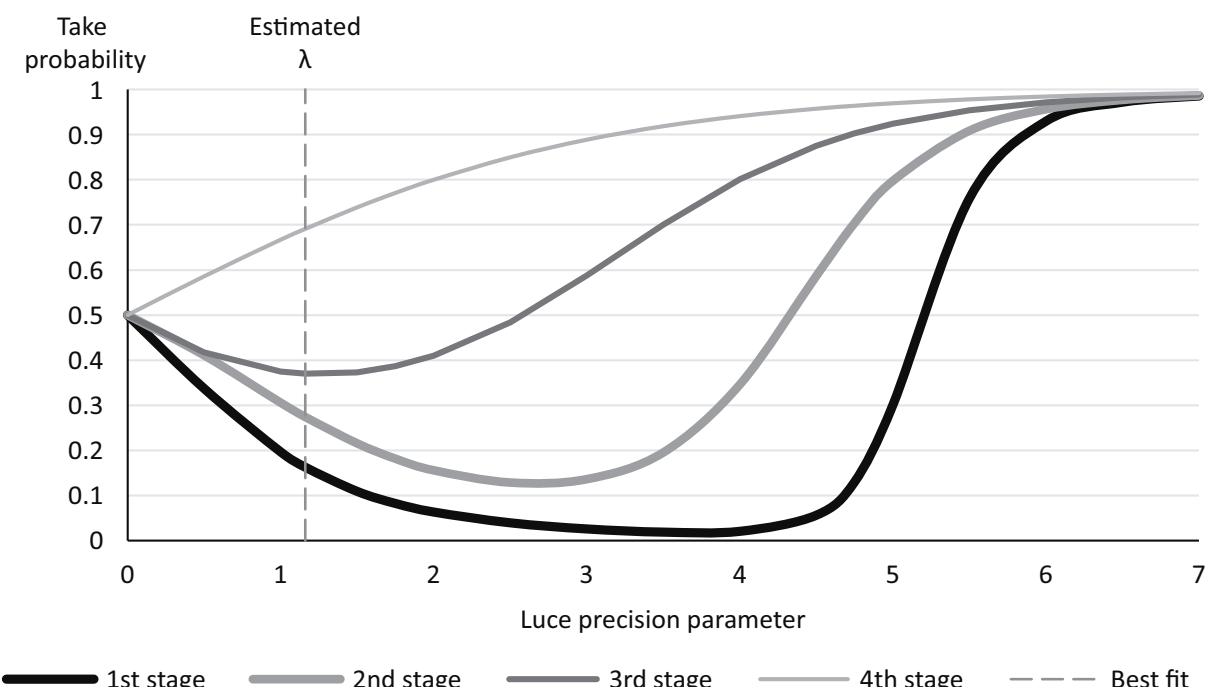

Notes: With a precision of 0 on the left-hand side, the take probabilities are all 0.5 (perfectly random). For each positive precision value shown on the horizontal axis, the take probabilities increase from stage 1 (darkest line) to stage 4 (lightest line). A Luce precision parameter of $\lambda=1.16$, as indicated by the vertical dashed line, generates the best-fit take probabilities provided in the bottom part of Table 1.1 for this four-stage game.

Figure 1.14 Luce QRE correspondence of take probabilities for the four-stage centipede game 
and six-stage games together requires an additional six intersection points to be arrayed on the same vertical line, which is generally impossible. The 10 fitted Luce predictions shown in the bottom rows of Table 1.1 (based on a single precision parameter) are not perfect, but they do track the key qualitative features of deviations from the Nash prediction of take rates of 1 at all stages.

\section{GAMES WITH A CONTINUUM OF DECISIONS: IMPERFECT PRICE COMPETITION}

Recall that a logit model is based on a binary choice probability expressed as a ratio of exponentials of expected payoffs, with $e^{\lambda \pi_{i}(p)}$ for decision $i$ in the numerator, and with a sum of analogous exponentials for both decisions in the denominator. With a larger number of decisions, the denominator sum includes exponentials for each decision, to ensure that the resulting choice probabilities sum to 1 . With a continuum of decisions on a range, say 0 to 100 , the probabilities are densities, $f(p)$, and the sum in the denominator is replaced with an integral:

$$
f(p)=\frac{e^{\lambda \pi(p)}}{\int_{0}^{100} e^{\lambda \pi\left(p^{\prime}\right)} d p^{\prime}}
$$

The next example illustrates a key insight about QRE as an equilibrium theory, and the effects of noise are not simply to spread decisions out around some central tendency. There can be cascading feedback effects that push the distributions of decisions far away from a Nash equilibrium that would occur in the absence of noise. Capra et al. (2002) report an experiment for a price competition game in which the unique Nash equilibrium was at the low end of the range of possible prices, but the data average for one of the treatments turned out to be about 70 percent of the way towards the high end of the price range. The reader might wonder how QRE can explain this pattern, since in some previous applications the effect of noise has been to pull the QRE predictions towards the center. In those games, the expected payoff difference line generally had a negative slope, for example, it is better not to volunteer when the others volunteer with high probability. In contrast, the payoff difference line for the price competition game, to be considered next, has a positive slope, that is, increased cooperation stimulates additional cooperation. In particular, when others tend to charge higher prices, a firm can earn more by raising the price, so there can be a cascading upward pressure on prices owing to equilibrium interaction effects.

When there are many possible decisions, for example, prices in pennies, it is convenient to model the game as having a continuum of choices. For example, suppose that there are two firms that are constrained to choose prices in a range from 60 to 160. Buyers will demand a total of $1+\alpha$ units of the product for prices in this interval, with the sales of the low-price firm normalized to be 1 , and the sales of the high-price firm being $\alpha<1$. There were two treatments in the experiment, one very competitive, with $\alpha=0.2$, and the other much less competitive, with $\alpha=0.8$. In this less competitive treatment, the high-price firm sells almost as much as the low-price firm. Prices are chosen independently, but the high-price firm must match that of the low-price firm ex post, for example, in a meet-or-release contract. The delay owing to the ex post price reduction is assumed to lower the sales quantity for the high-price firm. If prices are 90 and 100, for example, the firm with the 90 price sells 1 unit, and the other 
firm only sells a fraction $\alpha$ after matching the low price of 90 . Since $\alpha<1$, the low-price firm makes more money. It follows that each firm has an incentive to undercut the other, so the unique Nash equilibrium is at the minimum feasible price of 60 . Basic intuition, however, suggests that prices would be higher in the less competitive treatment.

This intuition was confirmed in a laboratory experiment that implemented the imperfect competition game with zero costs, with a series of random matchings between subjects acting as sellers. For the competitive treatment in which the high-price seller only sells a fifth as many units as the low-priced seller, the average price was 68 , close to the Nash equilibrium of 60 . However, for the less competitive treatment $(\alpha=0.8)$, the average price was 121 , almost twice as high as the Nash prediction, and on the other side of the midpoint of the price range. The cumulative distributions of observed prices, shown as sequences of dots in Figure 1.15, reveal a treatment difference that diverges from the sharp (dashed-line) Nash prediction for both treatments that puts all probability at the lowest feasible price.

A possible reaction to the high prices relative to the Nash equilibrium in the less competitive treatment is that it is just behavioral, a comment that we have heard on occasion. We used to be content with hanging this label on unexpected data patterns, but QRE offers a more general, theory-based approach that does not necessarily incorporate assumptions about behavioral biases. The first step in the analysis is to express the expected payoff function for each possible price choice $p$, given a belief density $f\left(p^{\prime}\right)$ that represents beliefs about the other's price $p^{\prime}$. With zero costs, this expected payoff is shown in equation (1.14), where the first term on the right-hand side is the price received for selling 1 unit times the probability that that price is the low price, where $F(p)$ is the cumulative of the belief density. The second term is the fractional

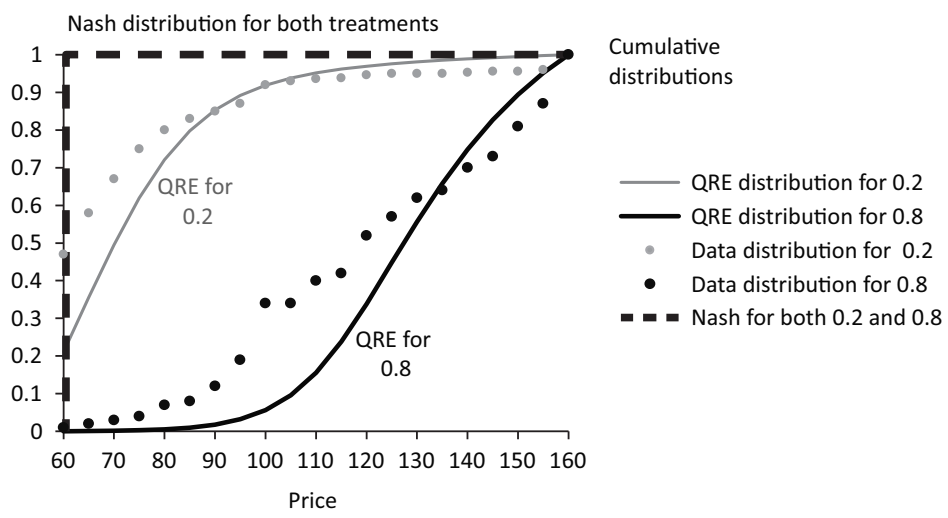

Notes: The two sequences of dots show cumulative data distributions for the two treatments in Capra et al. (2002). The dot sequence on the left is for the more competitive treatment $(\alpha=0.2)$, and the sequence on the right is for the less competitive treatment $(\alpha=0.8)$ that yields higher prices. The Nash equilibrium price prediction of 60 for both treatments produces a sharp cumulative dashed line that follows the left-hand and top sides of the figure. The smooth curved lines show the QRE cumulative distributions calculated using the experiment payoff parameters, but with a logit precision parameter that was previously estimated for a continuous-choice social dilemma experiment (Capra et al. 1999). The QRE densities can be used to calculate predicted price averages in a straightforward manner. In the less competitive treatment, the logit QRE price average is 128, at about the same level as the average price of 121 observed with human subjects in this treatment. In the more competitive treatment, the logit QRE price average is 78 , which is close to the observed data average of 68 .

Figure 1.15 Cumulative price distributions with imperfect price competition 
unit, $\alpha$, that is sold for each of the possible lower values of the other seller's price $p^{\prime}$, weighted by the belief density for $p^{\prime}$.

$$
\pi(p)=p[1-F(p)]+\alpha \int_{60}^{p} p^{\prime} f\left(p^{\prime}\right) d p^{\prime}
$$

In equilibrium, the choice density on the left side of (1.13) must be the same as the belief density that is used to determine the expected payoff function in (1.14). The solution to the resulting nonlinear equations involves finding a fixed point for a discretized distribution. The authors used a precision of $\lambda=0.12$, estimated previously for a different game, to solve for the equilibrium QRE distributions that are shown in Figure 1.15. ${ }^{16}$

Even though the discretized equilibrium computation involves finding a vector of probabilities that solve a large system of nonlinear equations, it is instructive to imagine a more mechanical, iterative approach. Consider a sequence of iterations, beginning with
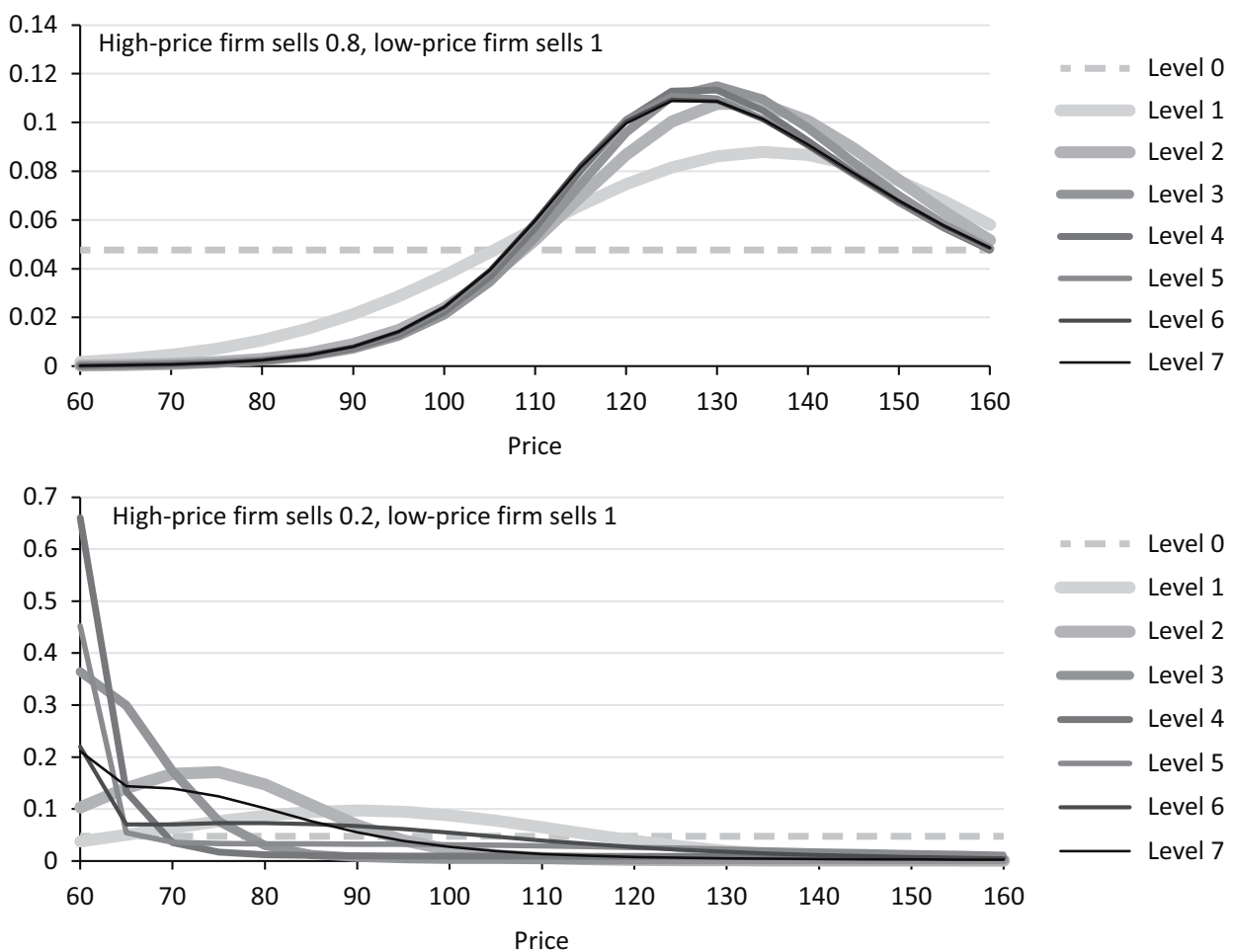

Notes: In each panel, an initial flat belief distribution (the horizontal dashed line) is used to calculate a logit quantal response density, which serves as the belief distribution for calculating a second logit quantal density, and so on. The iterated quantal responses converge to the QRE density in the top panel, with a peak at relatively high prices for this less competitive treatment. For the more competitive treatment, the analogous sequence of logit response densities tends to pile up near the low end of the price scale.

Figure 1.16 Iterated logit quantal responses with less severe price competition (top panel) or more price competition (bottom panel) 
a flat (uniform) belief distribution on the price interval $[60,160]$ that is used to calculate expected payoffs and a resulting probabilistic (logit) response density. That response density is then used as a belief distribution to generate a new logit choice distribution, etc. Figure 1.16 shows such a sequence of noisy quantal responses to the initial flat beliefs (the dashed gray horizontal line), using the precision parameter $\lambda$ estimated previously. Notice that the sequence of densities is converging after several iterations. The limit to this sequence is a quantal response equilibrium distribution, that is, a distribution that is mapped into itself (beliefs match choice distributions).

The flat beliefs used to start the iterative sequences in Figure 1.16 are sometimes referred to as level 0 beliefs. A level 1 player, who believes that other decisions are pure noise, would have a quantal response that will be termed noisy level 1 . A level 2 player is one who believes that others are noisy level 1 , and so on. The distributions at each level are not equilibrium distributions, since the surprise differences between choice and belief distributions would generate learning and a tendency to change. Here we see the relationship between QRE (as a fixed point limit) and noisy quantal responses to lower-level beliefs.

\section{Empirical Content of QRE in Models with a Range of Decisions}

As the logit precision goes to 0 , the probabilities collapse to a uniform level, so the average price prediction would be at the midpoint of the range of possible prices. As precision increases, the price averages move around in the range of possible decisions. In the imperfect price competition model, for example, average prices first increase and later decrease towards the Nash prediction at the lower bound as precision goes to infinity. Thus it could be possible to find a precision with an associated average QRE price prediction that exactly matches any observed data average in a wide range of prices. This possibility fades with the inclusion of a second treatment. Moreover, maximum likelihood estimates of the precision parameter are sensitive to the whole price distribution (a product of price probabilities, each raised to a power that equals the number of times that particular price is observed in the data). The resulting fit is never perfect. The only hope of getting a perfect fit for each of the cumulative distribution dots shown in Figure 1.15 would be to specify a separate precision for each possible price choice on $[60,160]$ for each treatment, for a total of over 200 parameters to be estimated. In contrast, the predictions shown in the figure are for a single parameter that was estimated previously using data for a different (social dilemma) game.

\section{NON-EQUILIBRIUM BELIEFS: LEARNING AND INTROSPECTION}

As with any equilibrium theory, one possible critique of QRE is that equilibrium may not be behaviorally plausible if players have not had a chance to learn about others' decisions from past plays of the same type of game with similar groups of other players. One experimentalist remarked: 'I like the QR but not the E.' Many situations involving political or legal conflict are so novel that players may have little or no information about actions chosen previously by others in similar settings. For example, it is known that players who tend to overestimate the cooperativeness of others tend to cooperate more in prisoner's dilemmas. In such settings, players must learn by introspection about what the other players might do, what others think that others might do, and so on. In other settings, learning based on prior observations is 
likely to be incomplete owing to short histories, imperfect recall or changing conditions. In either instance, with incomplete learning from past observations or noisy learning from introspection, belief distributions should not be required to exactly match action distributions. Nevertheless, models of introspection and learning from experience can incorporate smoothed quantal responses, even though beliefs are not pinned down by an equilibrium condition. ${ }^{17}$ In contrast, equilibrium models can be useful and accurate when players have had a chance to learn and adjust, as many of the experiments summarized above indicate. The point is that determining the appropriate model - QRE, quantal response learning or quantal response introspection - may depend on the novelty or stationarity of the situation being studied (Goeree and Holt 1999). In this section, we consider introspection and learning in the context of the imperfect price competition game.

\section{Level $k$ and Introspection}

First, consider the similarities and differences between QRE and introspection models based on iterated responses, commonly known as level- $k$ thinking. For readers who have not encountered this approach, you should think of level $k$ as a model of levels of strategic sophistication, where level 0 is totally noisy, choosing among all decisions with equal probability. So if prices could be between 60 and 160, for example, a level 0 person would select each price in that interval with equal probability, and their average price would be at the midpoint of 110. A level 1 person would make the best response to the uniform price distribution of a level 0 person, a level 2 person is thinking one step ahead and chooses a best response to the prices used by a level 1 person, and so on. The responses used in this chain are typically taken to be best responses with no noise, but it is natural to consider quantal responses determined by a relevant precision, as in the previous section in the discussion of the sequence of convergent curved lines in Figure 1.16. Since there is usually some variation in data collected in laboratory experiments, it is often desirable to consider probabilistic (quantal) responses to beliefs determined by level- $k$ analysis. This approach will be referred to as a quantal level- $k$ model.

The level- $k$ approach is best suited to modeling behavior in a game that is played only once, so all learning must be by introspection (although level- $k$ learning models will be discussed below). The standard level- $k$ implementation is commonly based on noise-free best responses that maximize expected payoffs (Stahl and Wilson 1994; Nagel 1995). For the imperfect competition game, it is straightforward to use a flat distribution over the 101 possible prices on the $[60,160]$ interval of feasible prices as a level 0 belief in order to calculate the profit maximizing price response. This level 1 best response turns out to be a price of 90 for the more competitive (low $\alpha$ ) treatment and 133 for the less competitive (high $\alpha$ ) treatment. The best response to a price of 133 is 132 , and the best response to 132 is 131 . Therefore, the level- $k$ best response predictions for levels 1,2, and 3 are 133, 132 and 131 for the less competitive treatment, and 90, 89 and 88 for the more competitive treatment. These level- $k$ best response predictions for each treatment are shown as clusters of adjacent thin horizontal dashed lines in Figure 1.17. ${ }^{18}$ As indicated by Figure 1.16, the quantal level- $k$ predictions would exhibit more spread, with predictions for higher levels being closer to the Nash prediction.

The level- $k$ model with best responses does a good job of predicting data (large dots in Figure 1.17) in the first round, especially for the more competitive treatment with prices that begin at about 90 . However, level $k$ is less useful for evaluating data averages over all rounds, 


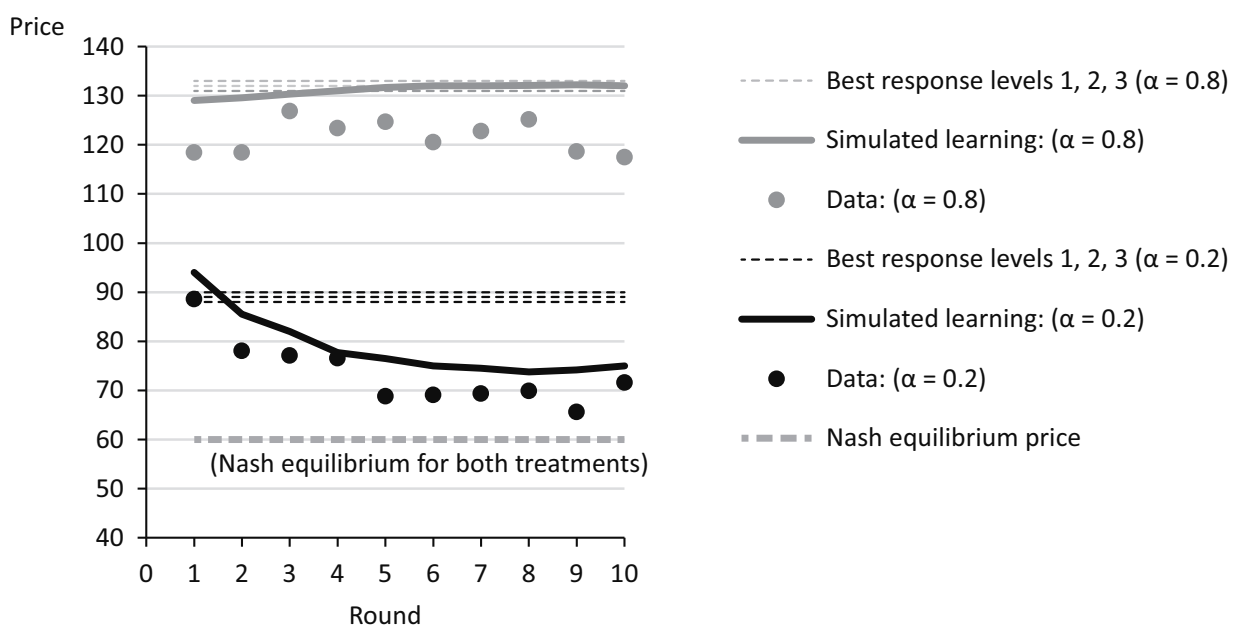

Notes: The sequence of price averages from Capra et al. (2002) is shown as the series of circular dots, for the high buyer inertia treatment $(\alpha=0.8)$ at the top and for the low inertia treatment $(\alpha=0.2)$ at the bottom. The level- $k$ best-response predictions for levels 1-3 (thin horizontal dashed lines) are 133, 132 and 131 for the high inertia treatment and 90, 89 and 88 for the low inertia treatments. Level- $k$ predictions are close to the data in the initial round, which is closest to the one-shot setting that level- $k$ is primarily designed for. However, these predictions are off the mark for the competitive low-inertia treatment as prices fall in later rounds, and as players learn and respond to other's price cuts. The qualitative features of the price trajectories are tracked by a simulated learning model (curved solid lines). The Nash equilibrium price is 60 for both treatments. The logit QRE predictions based on a precision estimated in a previous experiment are 128 for the less competitive (high $\alpha$ ) treatment and 78 for the less competitive (low $\alpha$ ) treatment, so QRE predicts (out of sample) the sharp deviations from the Nash prediction in one treatment and not in the other.

\section{Figure 1.17 Imperfect competition with simulated learning and level-k predictions}

since it takes no account of learning that would occur in a series of random matchings that generates a price history for each person. This learning was especially apparent in the more competitive $(\alpha=0.2)$ treatment, for which the average observed price fell from 88 in the initial period (at the level 1-3 predictions) to much more competitive price levels as price-cutting became prevalent.

\section{Belief Learning}

Models of learning are especially useful in explaining patterns of adjustment toward equilibrium, for example, whether prices tend to converge from above or below. For the model of imperfect price competition, the prices in the less competitive treatment started high, well away from the Nash prediction, and stayed high. In contrast, the observed prices for the more competitive treatment started in an intermediate range and fell continuously toward a level of about 70. This difference in adjustment patterns, with more movement from round to round in the more competitive treatment, is picked up by a simple learning model discussed in Capra et al (2002). Belief probabilities associated with each possible price are determined by a weight associated with that price, divided by the sum of the belief weights for all prices. This division ensures that the normalized weights are probabilities that sum to 1 (Goeree and Holt 2003b). The learning model begins with flat beliefs that result from equal weights for all 
prices, that is, a uniform distribution. When a particular price is observed, then the weight for that price (or price interval) is incremented, and the other weights are degraded in a manner ensuring that the sum of belief probabilities is 1 . This belief-learning model was used in simulations to explain observed patterns of price adjustment, that is, the tendency for observed prices to start high and stay high in the less competitive treatment, and for prices to decline toward Nash levels in the more competitive treatment. The learning model used precision and recency parameters taken from an earlier paper, Capra et al. (1999), to simulate a learning process with 12 subjects being randomly matched. The set of simulated price trajectories was averaged to generate learning model predictions, which are graphed as the curved solid lines for each treatment in Figure 1.17.

Belief-learning models are backward-looking in the sense that players are assumed to respond (stochastically) to prior observations. A more forward-looking, strategic approach could be to add a level of strategic thinking in that a player could choose a (stochastic) response to the anticipated stochastic responses of others to prior observations (Stahl and Wilson 1995). Breitmoser (2012) considers data from six different guessing-game studies, each with repeated interactions and target that was $2 / 3$ of the average guess. He concludes that the data are best explained by models that include some elements of stochastic choice and strategic thinking, that is, logistic level- $k$, noisy introspection (Goeree and Holt 2004) and QRE. ${ }^{19}$

In summary, the imperfect price competition experiment illustrates three distinct components of behavioral game theory: (1) level $k$ and noisy introspection models used to predict initial decisions in the absence of learning, (2) learning models that can be used to explain patterns of adjustment, and (3) equilibrium theories, such as QRE, that provide predictions of overall decision averages in equilibrium. The main message from Figures 1.16 and 1.17 is to clarify the distinctions between level- $k$ best responses, levels of quantal responses and a QRE that is a distribution that gets mapped into itself, which can sometimes (but not always) be approximated by the limit of an iterated series of quantal responses. The power of QRE arises from equilibrium restrictions that are analogous to the rational expectations conditions that pervade many macroeconomic models.

\section{HETEROGENEITY}

The random disturbance shocks being modeled with QRE result in a predicted distribution of decisions across individuals, even if individuals are otherwise identical. An additional source of heterogeneity can arise owing to variations in individual skills, preferences or opportunities. The simplest quantal response models can be generalized to allow error/precision rates to differ across individuals. This could reflect a number of idiosyncratic factors, such as differing levels of strategic sophistication, or rational inattention with individual variations in attention costs, abilities, and so on. A general model of this type is specified in Rogers et al. (2009), named heterogeneous quantal response equilibrium (HQRE). This approach also allows for different beliefs individuals have about the precision of the other players' decisions. Weizsäcker (2003) and McKelvey et al. (2000) consider these models, and there is some evidence that individuals underestimate the sophistication of others. ${ }^{20}$

A second source of heterogeneity can arise in the form of diverse preferences or biases. For example, individuals may differ in their risk attitudes or in some aspect of social preferences, such as inequality aversion, warm glow from socially beneficial behavior or altruism. Several 
studies report significant individual differences of this type. Palfrey and Prisbrey (1997) use a QRE structural model to estimate individual fixed effects in the form of warm glow and altruism in a voluntary contributions game. While behavior on average is close the Nash equilibrium, there is a wide variance in the individual choices, and they find significant effects of heterogeneity of a warm glow parameter, but not altruism. ${ }^{21}$ Using an alternative experimental design to measure altruistic behavior, Goeree et al. (2002a) apply QRE as a structural model to estimate a two-parameter random effects model of individual altruism parameters as i.i.d. draws from a truncated normal distribution with mean, $\mu$, and variance, $\sigma$. Finally, Goeree and Holt (2000) augment a QRE analysis of a two-stage bargaining experiment with the estimation of envy and greed parameters for a prominent behavioral model of inequity aversion (Fehr and Schmidt 1999).

Even the absence of persistent individual differences, a panel of people who make decisions in a sequence of $n$ trials with a probability of $p$ associated with decision $D 1$ would exhibit a binomial variance of $n p(1-p)$ across people. Goeree et al. (2017) report that variances in observed volunteer rates were an order of magnitude higher than would be implied by the binomial variance formula, which suggests clear differences in volunteer rates across subjects. In contrast, the fitted three-parameter $(\lambda, \mu$ and $\sigma)$ HQRE model yields predicted variances in volunteer rates across people that were similar to the variances observed in the data. Even though a simple QRE model did a reasonable job of fitting average volunteer rates across the six treatments, the HQRE did much better in explaining the variances. However, generalizations with additional parameters are only appropriate for data sets that span a range of different treatments, in order to avoid overfitting. Anyway, estimation involves the specification of an error structure, which is a natural component of QRE.

\section{Quantal Response Equilibrium for Bayesian Games}

Next consider a class of models in which individual differences are represented as random draws from a population distribution. This Bayesian game approach extends the formal structure of games in strategic form by adding two additional components: the set of type profiles, $T=T_{1} \times \cdots \times T_{N}$ and the set of player beliefs, where player $i$ 's belief about the profile of other players' types is denoted $\rho\left(t_{-i} \mid t_{i}\right)$. For player $i$ with available actions, $\mathrm{A}_{i}$, a (behavioral) strategy for $i, \sigma_{i}$, specifies a probability distribution over $\mathrm{A}_{i}$ as a function of $t_{i}$. A Bayes-Nash equilibrium of the game, $\sigma^{*}$, is a strategy profile with the property that each $i$ and for each type $t_{i}, \sigma_{i}^{*}\left(t_{i}\right)$ is optimal for $i$, given the strategies of the other players and given $i$ 's beliefs $\rho\left(t_{-i} \mid t_{i}\right)$.

A Bayesian QRE is defined similarly, except $\sigma_{i}^{*}\left(t_{i}\right)$ is a quantal response for $i$, given the strategies of the other players and given $i$ 's beliefs $\rho\left(t_{-i} \mid t_{i}\right)$. We illustrate it here with a Bayesian game version of the volunteer's dilemma discussed in section 4 . The only change is that the cost of volunteering is no longer assumed to be identical across players. Instead, each player has a privately known cost or type, denoted by $c_{i}$, with each private cost being an independent draw from a commonly known uniform distribution, $G(c)$ on the interval $\left[0, C_{\text {max }}\right]$. The i.i.d. nature of the draws implies that beliefs $\rho\left(t_{-i} \mid t_{i}\right)$ are independent of $t_{i}$ in this example. A strategy for player $i$ is a mapping from $\left[0, C_{\max }\right]$ into $[0,1]$. That is, a strategy specifies a probability of volunteering for each realized private cost, $c_{i}$. We limit the analysis here to symmetric Bayesian Nash equilibria, where all players use the same mapping. 
The Bayesian Nash equilibrium of the game is similar to a mixed Nash equilibrium of the game of complete information, except in the Bayesian Nash equilibrium, every type of every player actually adopts a pure strategy. The equilibrium divides the type space, $\left[0, C_{\max }\right]$ into low-cost types who volunteer with probability 1 and high-cost types who volunteer with probability 0 , with a critical cost, $c^{*}$, defining the cutoff between the volunteering types and free-rider types. In equilibrium, the cutoff type is indifferent between volunteering and free riding. Thus, a symmetric equilibrium of the game is determined by equating the cutoff cost with the expected benefit of volunteering, which is the value of getting at least one volunteer, $V$, times the pivotal probability that none of the $N-1$ other players volunteer:

$$
c^{*}=V\left(1-P^{*}\right)^{N-1},
$$

where $P^{*}=G\left(c^{*}\right)$ is the equilibrium probability that a player is a volunteering type, that is, the probability that a random cost realization is below the cutoff $c^{*}$.

The private information and heterogeneity in the Bayesian game generally leads to Nash equilibrium volunteer rates that differ from the volunteer rate in the complete information version of the game if everyone simply had the same cost equal to the expected value of the random cost draws. To illustrate this difference, consider the same parameters used for the complete information volunteer's dilemma game in section 4 , where $V=0.8$ and $C=0.2$. With two players, the mixed strategy Nash equilibrium volunteer rate was $p^{*}=3 / 4$ (shown as a diamond at 0.75 on the vertical line in Figure 1.6). Compare this with the Bayesian Nash equilibrium of the game where the distribution of cost draws, $G$, is uniform on [0, 0.4]. In that case, $P^{*}=G\left(c^{*}\right)=c^{*} / 0.4$ or, equivalently, $c^{*}=0.4 P^{*}$. Plugging this into the equilibrium equation (1.15), gives $P^{*}=2 / 3$, which differs from the Nash mixed-strategy equilibrium volunteer probability of $3 / 4$ with two players and a deterministic cost $(C=0.2)$ at the midpoint of the range of random cost draws in the Bayesian game.

A symmetric logit QRE of the Bayesian game assigns a volunteer probability to each possible cost type, $p(c)$, which satisfies the logit equation:

$$
p(c)=\frac{1}{1+\exp \left(-\lambda\left[V\left(1-P^{*}\right)^{N-1}-c\right]\right)}
$$

where $P^{*}=\int_{0}^{C m a x} p(c) d G(c)$ is the expected probability that another player volunteers. That is, the logit QRE smooths out the step function cutoff strategy, so higher-cost types are less likely to contribute than lower-cost types, but no type volunteers with probability 0 or probability 1 . It is not difficult to write a simple program to compute the logit QRE volunteer rates for this game as a function of $\lambda, N, V$ and $C$. These calculations permit a comparison of QRE volunteer rates for the Bayesian game, where costs are drawn from the interval $[0,0.4]$ and the complete information game with a fixed cost of 0.2 for all players. While the exact volunteer probabilities are different in complete and incomplete information formulations, they share the same qualitative features, but with the familiar pull-to-center QRE effect; namely, the QRE volunteer rate is less than the (Bayes) Nash equilibrium if the (Bayes) Nash equilibrium is greater than 0.5. Conversely, the QRE volunteer rate is greater than the Bayes-Nash equilibrium if that equilibrium is below 0.5. Furthermore, for $N>3$, it can be shown that the success probability is increasing in $N$ and converges to 1 , for every 
fixed value of $\lambda$ (details available on request). Moreover, the empirical content of QRE for this model is just as strong as for the complete information volunteer's dilemma, since in each case there is a single precision parameter that is used to derive volunteer rate predictions for a wide range of group size treatments.

\section{SUMMARY}

This chapter uses a series of increasingly complex games to show how QRE can be represented by decomposing expected payoffs and cumulative distributions of payoff disturbances. The graphical analysis of symmetric games with binary decisions shows that a standard assumption of i.i.d disturbances ensures that the cumulative distribution of the disturbance differences will equal 0.5 at a difference of 0 . This symmetry property is implemented in virtually all empirical tests of quantal response (logit, probit, and so on), and it provides clear empirical content for QRE predictions. For several of the social dilemma games considered, the QRE predictions are pulled to the center, which implies that choice probabilities that are more extreme than the mixed-strategy Nash predictions are ruled out for all possible values of the logit or probit precision parameter. In contrast, the minimum-effort coordination game yields predictions that are pulled to the extremes relative to a mixed Nash equilibrium, which rules out any choice proportions between the Nash prediction and 0.5 . In either instance, empirical content is further enhanced by using a single precision estimate for multiple treatments, for games with wide ranges of decisions or for generating predictions for different data sets. Alternatively, empirical content is weakened by adding parameters, for example, means and variances of distributions of individual effects or propensities, as with any applied work intended to explain individual heterogeneity. Quantal response does have clear empirical content in that there are ranges of choice proportions that cannot be explained with any estimated precision in some very simple games, even with a single treatment and only two possible decisions.

Quantal response equilibrium represents a generalization of standard game theory, with a unified structure that permits the study of introspection, learning and equilibrium. Moreover, the statistical nature of QRE offers a natural framework for structural estimation of behavioral parameters, in a setting where noise can have interacting equilibrium effects. Any estimation requires a disturbance component, regardless of whether the data arise from individual decisions or from interactive games. With games, it is reasonable to build the disturbances into the structure of the interactions, instead of just appending an error on to observed data. Since QRE is a statistical model that assigns positive probabilities (however small) to all feasible actions, it permits estimation in a natural manner. The resulting equilibrium statistical models can be used to incorporate insights and biases that are documented in research on behavioral economics, for example, the effects of inequity aversion, regret, altruism or risk aversion in auctions and games. ${ }^{22}$ Quantal response equilibrium has also been applied to sequential games (for example, McKelvey and Palfrey 1992, 1998; Sieberg et al. 2013), where quantal continuation values are used to calculate equilibria that exhibit a generalized version of subgame perfection. These quantal continuation values make it possible to look 'inside the box' and gain an understanding of the underlying process that generates intuitive but systematic departures from theoretical predictions, departures that arise naturally from intersections or interactions of smoothed quantal responses that replace sharp corners implicit in standard models with perfect rationality. ${ }^{23}$ 
It is common for traditional economists to view the variety of biases that have emerged from the behavioral economics literature with bewilderment, given that each bias has been tailormade to explain one or more seemingly anomalous findings. Quantal response equilibrium provides a coherent theory for addressing many different behavioral anomalies within a common framework, yet capable of incorporating and measuring the effects of unobserved behavioral variables and/or bounded rationality. This theory has produced dramatic results in that seemingly anomalous (but intuitive) deviations from standard theory are often well aligned with QRE predictions. In summary, the smoothed QRE models can shine a light on the underlying structure of otherwise confusing data arrays and thereby enhance the behavioral relevance of game theory as it is increasingly used in social sciences.

\section{NOTES}

* This research was funded in part by the University of Virginia Quantitative Collaborative and the National Science Foundation (Holt: NSF-1459918 and Palfrey: SES-1426560) and the Australian Research Council (Goeree: DP150104491). We benefitted from suggestions by Michelle Song, Nicholas Anderson, Georgia Beazley, Ritika Gupta, Madison Smither, Yang Yu, members of the University of Virginia Quantitative Collaborative and participants in the University of Pittsburgh Experimental Lab Seminar.

1. Recall that in non-cooperative games, adding a constant to all payoffs has no strategic consequences, in that it does not change best response functions and does not change the set of Nash equilibria or correlated equilibria of the games.

2. We can include a precision parameter for any generic specification of a quantal response function. For example, the power version of the Luce ratio rule raises expected payoffs to a power $\lambda: \operatorname{Pr}(D 1 ; \lambda)=\pi_{1}^{\lambda} /\left(\pi_{1}^{\lambda}+\pi_{2}^{\lambda}\right)$.

3. Some quantal response equilibria can be rationalized as Bayesian equilibria with additive privately known payoff disturbances; see McKelvey and Palfrey $(1995,1998)$. However, some QREs cannot be rationalized as such, for example, QRE based on the Luce ratio or more general, regular quantal response functions (Goeree et al. 2005).

4. About 65 years ago, John Nash's thesis advisor saw the payoffs for this type of game on the blackboard of a colleague's office at RAND in Santa Monica, and he made up the story of the prisoner's dilemma for a presentation in the Stanford psychology department on recent developments in game theory. The payoffs, however, had been devised by two RAND researchers on the day they heard about Nash's famous theorem on equilibrium in non-cooperative games, and these payoffs were used in an experiment involving over 100 repeated plays of this game with the same partner. In a letter to the authors, John Nash later noted that the theory for a single-shot game might not be applicable with repetition. For details, see Holt and Roth (2004).

5. All Nash equilibria of the centipede game involve taking at the first stage, so this is more than just a violation of backward induction. This is in stark contrast with the ultimatum game, where every possible offer is consistent with Nash equilibrium.

6. Since an individual decision problem is a trivial special case of a game, this observation by Haile et al. (2008) also applies to standard probabilistic choice models (logit or probit) that are extensively used in applied econometric work. Obviously, the critique is not relevant in that case either, since the i.i.d. assumption is standard in applied econometric work on individual decisions, just as it is standard in the application of QRE to the statistical analysis of game-theoretic data.

7. This conclusion is derived more formally in Goeree et al. (2005, 2016, chs 2 and 6). The notion of a regular QRE, as defined in Goeree et al. (2005), is grounded on axioms of a responsiveness, monotonicity and continuity that preserve strong empirical restrictions, including those implied by i.i.d. payoff disturbances.

8. A clear understanding of the strategic landscape of the games to be discussed can be achieved if they are run as class experiments. See Holt (2019, chs 8-11) for hints on how to use the free web-based Veconlab site for this purpose (http://veconlab.econ.virginia.edu/admin.php, accessed 12 July 2020). An alternative is to use the popular Moblab platform (https://www.moblab.com/, accessed 12 July 2020), which also has games with a political science focus.

9. Sample Matlab code for such calculations is provided in Goeree et al. (2016, ch. 6).

10. This decomposition was used in Goeree and Holt (2005b) for an analysis of political participation games with binary decisions, for example, vote or not. The difference here is that the probability is shown on the vertical axis, so that the inverse distribution has the familiar shape of a cumulative distribution function.

11. This observation is roughly consistent with the 10-20 percent cooperation rates (except for the first and last periods) observed by Andreoni and Miller (1993) with repeated random matching (strangers). Cooperation rates 


\section{Handbook of experimental game theory}

were higher in a finitely repeated prisoner's dilemma with the same payoffs and with fixed pairings (partners). The QRE analysis for repeated games is more involved (Goeree et al. 2016, ch. 4). In addition to repetition, another aspect of many strategic interactions in business and politics is the ability to choose our partners and terminate pairings that are unsatisfactory, or even to exit and earn a default payoff for no pairing at all. See Holt et al. (2015) for a general discussion of prisoner's dilemma games with the ability to exit and/or scale up the intensity of interactions, factors that produce dramatically higher cooperation rates even in a finite series of random matchings, and hence, no ability to terminate pairings.

12. This procedure is justified by inverting $x=\Delta(p)$ to get $p=\Delta^{-1}(x)$ and then substituting this into (3) to get $\Delta^{-1}(x)$ on the left-hand side and $F(\lambda \Delta(p))=F\left(\lambda \Delta\left(\Delta^{-1}(x)\right)\right)=F(\lambda x)=1 /(1+\exp (-\lambda x))$ on the right-hand side.

13. To verify this unintuitive property, set the payoff difference equal to zero and solve for $(1-p)^{N}$ to obtain $(1-p)^{N}=(C / V)^{N /(N-1)}$ which is increasing in $N$, with a limiting value of $C / V$.

14. Zauner used a probit quantal response function based on normally distributed payoff disturbances, while McKelvey and Palfrey used the logit quantal response function.

15. This rule, which requires that payoffs be non-negative, can be derived by assuming that random payoff perturbations are multiplicative with mean 1, instead of additive with mean 0 (Goeree et al. 2016, ch. 1). With multiplicative disturbances, decision 1 is selected if $\pi_{1} \varepsilon_{1 j}{ }^{1 / \lambda}>\pi_{2} \varepsilon_{2 j}{ }^{1 / \lambda}$, where a high $\lambda$ results in essentially no noise, and the disturbances perturbations are non-negative and i.i.d. with median 1 . Then take the natural $\log$ of both sides of this inequality and multiply by $\lambda$. If the cumulative distribution of the difference in log disturbances is exponential, then the equation analogous to (1.5) involves exponentials of logs of power functions, which yields the ratio of power functions in (1.11).

16. In particular, the precision estimate was taken from a prior traveler's dilemma game experiment (Capra et al. 1999) with a different payoff structure, but with the same subject pool and random-matching protocol that was used subsequently in the imperfect price competition paper.

17. Quantal response learning and introspection models are developed and applied in Goeree et al. (2016, chs 5, 8 and 9).

18. There are many variations of iterated-thought models. Camerer et al. (2004) proposed a 'cognitive hierarchy' model in which a person of level $k$ assumes everyone else is of a lower level of rationality, so a level 2 person would view the world as being populated by level 0 and level 1 people. The truncated distributions of levels can be modeled with a Poisson distribution that assigns probabilities to each of the integers below one's own level. Alternatively, Goeree and Holt $(2001,2004)$ model noisy introspection of quantal responses to "he thinks, I think' layers of iterated beliefs, with more noise for higher numbers of iterations. This noisy introspection model was used to explain data patterns from a wide range of single-shot $2 \times 2$ games. Cabrera et al. (2007) also consider a noisy model of iterated conjectures, with conjectured decisions being drawn from a logit distribution, but after the draw, being treated as point beliefs with no uncertainty. A simple stopping rule is used to end the process when the circle is closed in that the initial conjecture about the other's decision matches the other's conjectured response to their own conjectured decision. The model was solved numerically and then used to explain data from a one-shot traveler's dilemma experiment.

19. It is natural to allow for subjects to exhibit various levels of strategic responses that include level 1, level 2, or higher levels that more closely approximate quantal response equilibrium play. To this end, Breitmoser et al. (2014) use data from a club game to study a steady state of different levels that evolves during repeated plays of this game.

20. For a detailed discussion of HQRE models, see Goeree et al. (2016, ch. 3).

21. For a related analysis using a similar voluntary contribution environment but a different subject pool, see Palfrey and Prisbrey (1996).

22. The Goeree et al. (2003) paper takes the safe and risky payoffs from the Holt and Laury (2002) price-list menu and incorporates those payoffs into a two-person, matching-pennies game. Then the joint estimation of risk aversion and QRE precision parameters is used to explain why one player will overplay the safe strategy relative to Nash, and the other player will not, a pattern that is observed in the data. The Goeree et al. (2002b) paper considers an auction market in which the Nash bidding strategies are the same for two treatments, but for which there is considerably more overbidding in the treatment where the losses from overbidding relative to Nash are lower, a pattern that is consistent with QRE predictions, augmented by risk aversion.

23. Also see Goeree et al. (2016, chs 3 and 8).

\section{REFERENCES}

Andreoni, J. and J.H. Miller (1993), 'Rational cooperation in the finitely repeated prisoner's dilemma: experimental evidence', Economic Journal, 103 (418), 570-85. 
Baron, D. and J. Ferejohn (1989), 'Bargaining in legislatures', American Political Science Review, 83 (4), 1181-206. Breitmoser, Y. (2012), 'Strategic reasoning in p-beauty contests', Games and Economic Behavior, 75 (2), $555-69$.

Breitmoser, Y., J.W.H. Tan and D.J. Zisso (2014), 'On the beliefs off the path: equilibrium refinement due to quantal response and level-k', Games and Economic Behavior, 86 (C), 102-25.

Cabrera, S., C.M. Capra and R. GÃşmez (2007), 'Behavior in one-shot traveler's dilemma games: model and experiments with advice', Spanish Economic Review, 9 (2), 129-52.

Camerer, C.F., T. Ho and J. Chung (2004), 'A cognitive hierarchy model of behavior in games', Quarterly Journal of Economics, 119 (3), 861-98.

Capra, C.M, J.K. Goeree, R. Gomez and C.A. Holt (1999), 'Anomalous behavior in traveler's dilemma', American Economic Review, 89 (3), 678-90.

Capra, C.M, J.K. Goeree, R. Gomez and C.A. Holt (2002), 'Learning and noisy behavior in an experimental study of imperfect price competition', International Economic Review, 43 (3), 613-36.

Cooper, R., D.V. DeJong, R. Forsythe and T.W. Ross (1989), 'Communication in the battle of the sexes game', Rand Journal of Economics, 20 (4), 568-87.

Cooper, R., D.V. DeJong, R. Forsythe and T.W. Ross (1996), 'Cooperation without reputation: experimental evidence from prisoner's dilemma experiments', Games and Economic Behavior, 12 (2), 187-218.

Fehr, E. and K.M. Schmidt (1999), 'A theory of fairness, competition, and cooperation', Quarterly Journal of Economics, 114 (3), 817-68.

Fudenberg, D. and D. Kreps (1993), 'Learning mixed equilibria,' Games and Economic Behavior, 5 (3), 365-82.

Goeree, J.K. and C.A. Holt (1999), 'Stochastic game theory: for playing games, not just for doing theory', Proceedings of the National Academy of Sciences of the USA, 96 (19), 10564-7.

Goeree, J.K. and C.A. Holt (2000), 'Asymmetric inequity aversion and noisy behavior in alternating offer bargaining games', European Economic Review, 44 (4-6), 1057-68.

Goeree, J.K. and C.A. Holt (2001), 'Ten little treasures of game theory and ten intuitive contradictions', American Economic Review, 91 (5), 1402-22.

Goeree, J.K. and C.A. Holt (2003a), 'Coordination games', in L. Nadel (ed.), Encyclopedia of Cognitive Science, vol. 2, London: Macmillan, pp. 204-8.

Goeree, J.K. and C.A. Holt (2003b), 'Learning in economics experiments', in L. Nadel (ed.), Encyclopedia of Cognitive Science, vol. 2, London: Macmillan, pp. 1060-69.

Goeree, J.K. and C.A. Holt (2004), 'A model of noisy introspection', Games and Economic Behavior, 46 (2), $365-82$.

Goeree, J.K. and C.A. Holt (2005a), 'An experimental study of costly coordination', Games and Economic Behavior, $51(2), 349-64$

Goeree, J.K. and C.A. Holt (2005b), 'An explanation of anomalous behavior in models of political participation', American Political Science Review, 99 (2), 201-13.

Goeree, J.K., C.A. Holt and S.K. Laury (2002a), 'Private costs and public benefits: unraveling the effects of altruism and noisy behaviour', Journal of Public Economics, 83 (2), 255-76.

Goeree, J.K., C.A. Holt and T.R. Palfrey (2002b), 'Quantal response equilibrium and overbidding in first-price auctions', Journal of Economic Theory, 104 (1), 247-72.

Goeree, J.K., C.A. Holt and T.R. Palfrey (2003), 'Risk averse behavior in generalized matching pennies games', Games and Economic Behavior, 45 (1), 97-113.

Goeree, J.K., C.A. Holt and T.R. Palfrey (2005), 'Regular quantal response equilibrium', Experimental Economics, $8(4), 347-67$.

Goeree, J.K., C.A. Holt and T.R. Palfrey (2016), Quantal Response Equilibrium: A Stochastic Theory of Games, Princeton, NJ: Princeton University Press.

Goeree, J.K., C.A. Holt and A. Smith (2017), 'An experimental examination of the volunteer's dilemma', Games and Economic Behavior, 102 (January), 303-15.

Goeree, J.K., C.A. Holt, P. Louis, T.R. Palfrey and B. Rogers (2019), 'Rank dependent choice equilibrium: a nonparametric generalization of QRE', in A. Schram and A. Ule (eds), The Handbook of Research Methods and Applications in Experimental Economics, Cheltenham, UK and Northampton, MA, USA: Edward Elgar, pp. 252-68.

Güth, W., R. Schmittberger and B. Schwarze (1982), 'An experimental study of ultimatum bargaining', Journal of Economic Behavior and Organization, 3 (4), 367-88.

Haile, P.A., A. Hortaçsu and G. Kosenok (2008), 'On the empirical content of quantal response equilibrium', American Economic Review, 98 (1), 180-200.

Harsanyi, J.C. (1973), 'Games with randomly distributed payoffs: a new rationale for mixed-strategy equilibrium points', International Journal of Game Theory, 2 (December), 1-23.

Holt, C.A. (2019), Markets, Games, and Strategic Behavior: A First Course in Experimental Economics, Princeton, NJ: Princeton University Press.

Holt, C.A. and S. Laury (2002), 'Risk aversion and incentive effects', American Economic Review, 92 (5), $1644-55$.

Holt, C.A. and T.R. Palfrey (2020), 'Binary conflict: theory and experiment', discussion paper, California Institute of Technology, Pasadena, CA. 
Holt, C.A. and A.E. Roth (2004), 'The Nash equilibrium, a perspective', Proceedings of the National Academy of Sciences of the USA,' 101 (12), 3999-4002.

Holt, C.A, C. Johnson and D. Schmidtz (2015), 'Prisoner's dilemma experiments', in M. Peterson (ed.), The Prisoner's Dilemma, Cambridge: Cambridge University Press, pp. 243-64.

Luce, R.D. (1959), Individual Choice Behavior, New York: Wiley.

McFadden, D. (1976), 'Quantal choice analysis: a survey', Annals of Economic and Social Measurement, 5 (4), 363-90.

McKelvey, R.D. and T.R. Palfrey (1992), 'An experimental study of the centipede game', Econometrica, 60 (4), 803-36.

McKelvey, R. and T.R. Palfrey (1995), 'Quantal response equilibrium', Games and Economic Behavior, 10 (1), 6-38.

McKelvey, R. and T.R. Palfrey (1996), 'A statistical theory of equilibrium in games', Japanese Economic Review, 47 (2), 186-209.

McKelvey, R.D. and T.R. Palfrey (1998), 'Quantal response equilibria for extensive form games', Experimental Economics, 1 (1), 9-41.

McKelvey, R.D. and T.R. Palfrey and R.A. Weber (2000), 'The effects of payoff magnitude and heterogeneity on behavior in $2 \times 2$ games with unique mixed strategy equilibria', Journal of Economic Behavior and Organization, 42 (4), 523-48.

Nagel, R. (1995), 'Unravelling in guessing games: an experimental study', American Economic Review, 85 (5), 1313-26.

Nash, J.F. (1950), 'Equilibrium points in N-person games', Proceedings of the National Academy of Science of the USA, $36(1), 48-9$.

Ochs, J. (1995), 'Coordination problems', in J.H. Kagel and A.E. Roth (eds), The Handbook of Experimental Economics, Princeton, NJ: Princeton University Press, pp. 195-249.

Ostrom, E. (1990), Governing the Commons: The Evolution of Institutions for Collective Action, Cambridge: Cambridge University Press.

Ostrom, E., R. Gardner and J.K. Walker (1994), Rules, Games, and Common-Pool Resources, Ann Arbor, MI: University of Michigan Press.

Palfrey, T. and J. Prisbrey (1996), 'Altruism, reputation, and noise in linear public goods experiments', Journal of Public Economics, 61 (3), 409-27.

Palfrey, T. and J. Prisbrey (1997), 'Anomalous behavior in linear public goods experiments: how much and why?', American Economic Review, 87 (5), 829-46.

Parco, J.E., A. Rapoport and W.E. Stein (2002), 'Effects of financial incentives on breakdown of mutual trust', Psychological Science, 13 (3), 292-7.

Rogers, B., T.R. Palfrey and C.F. Camerer (2009), 'Heterogeneous quantal response equilibrium', Journal of Economic Theory, 144 (4), 1440-67.

Rosenthal, R. (1982), 'Games of perfect information, predatory pricing, and the chain store paradox', Journal of Economic Theory, 25 (1), 92-100.

Roth, A.E. (1992), Two Sided Matching: A Study in Game-Theoretic Modeling and Analysis, Cambridge: Cambridge University Press.

Roth, A.E. (1995), 'Bargaining experiments', in J. Kagel and A. Roth (eds), Handbook of Experimental Economics, Princeton, NJ: Princeton University Press, pp. 253-348.

Selten, R. (1965), 'Spieltheoretische Behandlung eines Oligopolmodells mit Nachfragetragheit' ('Game theory treatment of an oligopoly model with sluggish demand'), Zeitschrift fãijr die gesamte Staatrwissenschaft, 121 (2), 301-24, 667-89.

Selten, R. and R. Stoecker (1986), 'End behavior in sequences of finite prisoner's dilemma supergames: a learning theory approach', Journal of Economic Behavior and Organization, 7 (1), 47-70.

Sieberg, K., D. Clark, C.A. Holt, T. Nordstrom and W. Reed (2013), "Experimental analysis of asymmetric power in conflict bargaining', Games and Economic Behavior, 4 (3), 375-97.

Stahl, D.O. and P.R. Wilson (1994), 'Experimental evidence on players' models of other players', Journal of Economic Behavior and Organization, 25 (3), 309-27.

Stahl, D.O. and P.R. Wilson (1995), 'On players' models of other players - theory and experimental evidence', Games and Economic Behavior, 10 (1), 213-54.

Svorenèík, A. and H. Mass (eds) (2016), The Making of Experimental Economics, Witness Seminar on the Emergence of a Field, New York: Springer.

Thurstone, L. (1927), 'A law of comparative judgment', Psychological Review, 34 (4), 273-86.

Tirole, J. (1988), The Theory of Industrial Organization, Cambridge, MA: MIT Press.

Van Huyck, J.B., R.C. Battalio and R.O. Beil (1990), 'Tacit coordination games, strategic uncertainty, and coordination failure', American Economic Review, 80 (1), 234-48.

Vickrey, W. (1961), 'Counterspeculation, auctions, and competitive sealed tenders', Journal of Finance, 16 (1), $8-37$. 
Von Neumann, J. and O. Morgenstern (1944), Theory of Games and Economic Behavior, Princeton, NJ: Princeton University Press.

Weizsäcker, G. (2003), 'Ignoring the rationality of others: evidence from experimental normal form games', Games and Economic Behavior, 44 (1), 145-71.

Zauner, K.G. (1999), 'A payoff uncertainty explanation of results in experimental centipede games', Games and Economic Behavior, 26 (1), 157-85. 